\title{
Multi-objective routing aware of mixed IoT traffic for low-cost wireless Backhauls
}

\author{
Vinícius N. Medeiros ${ }^{*}$ (D), Bruno Silvestre and Vinicius C. M. Borges
}

\begin{abstract}
The futuristic wireless networks expects to provide adequate support for distinct kind of applications, their diverse requirements, and scenarios for future Internet systems, such as Internet of Things based on multimedia and sensor data, while figuring out low cost solutions to offload the mobile communication core. In this context, Low-cost Wireless Backhauls (LWBs) can be useful, since they are based on cheap WLAN technologies, such as Wireless Mesh Networks that provide capacity for future loT applications based on mixed traffic. The routing is a fundamental process to provide communication in these multi-hop networks and multi-objective routing optimization algorithms based on Integer Linear Programming (ILP) models have been studied in the literature to address this problem, but there is a lack of solutions for mixed traffic. For this reason, we propose a novel ILP multi-objective approach, called Multi-objective routing Aware of miXed trafflc (MAXI), which employs three weighted objectives to guide the routing in WMNs with different applications and requirements. In addition, we provide a comparative analysis with other relevant approaches of routing using NS-3 to evaluation based on simulation, that takes into account different types and levels of interference (e.g. co-channel interference and external interference) focused on mixed loT traffic for elderly healthcare scenario. Finally, we demonstrate the effectiveness of the proposed approach to support the requirements of each application through the appropriate combination of objective functions, mainly in dense scenarios with high level of interference.
\end{abstract}

Keywords: Low-cost wireless Backhauls, Integer linear programming, Multi-objective routing, Mixed and multiple loT applications, Wireless mesh networks

\section{Introduction}

Internet of Things (IoT) has emerged as an important technology for smart environments, because it provides a set of operations (sensing, processing, and communication) combined in small devices. It will give rise to a vast number of applications, such as environmental monitoring [1-3], real time applications $[4,5]$, surveillance $[6]$, healthcare $[7,8]$, and agriculture $[9,10]$. These applications will include IoT applications based on general sensing data (low sending rates with or without a time restriction) and multimedia applications (video or virtual reality with high transmission rate demand mostly with delivery restriction) [11]. IoT devices are inexpensive and are being deployed and connected in large numbers and many public places to enable smart environments

*Correspondence: viniciusmedeiros@inf.ufg.br Instituto de Informática (INF) - UFG, Alameda Palmeiras, Quadra D, Câmpus Samambaia, 74690-900 Goiânia, Brazil based on a mixed of applications. Thus, there is a huge demand for coverage involving these low-power devices, the aggregate throughput demand, and reduce the end-toend delay.

The next generations of wireless/mobile networks should support a huge increase in the data traffic demand [12], diversity of performance requirements and applications (e.g. augmented reality, telemedicine, M2M/IoT, ultra-resolution videos) and to provide high ubiquity levels. Some new technologies [13] are being developing to achieve the ultra requirements, such as interference cancellation, LTE-U, FoG, Could-RAN, multi-access edge computing, millimeter wave, and massive MIMO. Besides, the futuristics wireless and mobile networks will also be under significant pressure to keep control of CAPEX and OPEX costs $[12,14,15]$, since mobile operators cannot count transfer the costs top the mobile users' bills to pay the development and deployment of these new technologies. In this context, the employment of cheap 
approaches is incentived, such as infrastructure and spectrum sharing [16] and heterogeneous networks composed of existing technologies $[17,18]$. For instance, Wi-Fi and ZigBee are the most used and cheap wireless technologies in heterogeneous networks [19].

In developing and big countries, such as Brazil, Mexico, India, and South Africa, there are a lot of poor and dense communities, which really needs low-cost mobile Internet access options, because they are not able to pay for mobile data plans based on LTE or millimeter wave technologies. Low-cost Wireless Backhauls (LWBs) are based on WiFi technologies and can be a method that enables mobile operator to extend their network access infrastructures to these communities, offering affordable mobile data plans. LWBs have made the Internet ubiquitous through some facilities such as the mobility, low-cost, and versatility of the equipment that is installed, which previously depended on fixed and network cable points.

Wireless Mesh Networks (WMNs) can be seen as a LWBs [20], since they are generally built with low-cost off-the-shelf hardware, such as Wi-Fi standards (IEEE 802.11a, $\mathrm{n}, \mathrm{b} / \mathrm{g})$. They provide a solution with a wider range (100 meters) and higher transmission rates (300 Mbps in the IEEE 802.11n standard), and can thus provide increased aggregate throughput through the use of multiple wireless network interfaces and multi-channel. WMNs are also a multi-hop communication networks, i.e., the information is forwarded (routed) by a mesh router until it reaches the Internet gateway, where it is processed or stored in the Internet [21, 22]. Moreover, the Wireless Sensor Networks (WSNs) can be connected to WMNs through the brigde technologies, for instance Raspberry $\mathrm{Pi}$. Thus, WMNs are a promising communication technology for IoT applications, because they provide a notably high performance and low cost wireless backbone for data in remote locations, when forwarding data from/to the Internet. Hence, WMNs and WSNs can be integrated to allow scenarios with a wide range of IoT applications, in order to reach balance between performance, cost, and ubiquity.

WMNs have a features that directly affect the performance of these networks, such as multi-hop communication, with a gateway for connection to other wireless or wired networks (by clustering generated data or sending data to these networks), as well as interference which restricts the useful capacity of these kinds of networks. In addition, other factors arise as a result of the multi-hop communication carried out by wireless networks, i.e. a) the path size that affects the contention level in the network and end-to-end delay (which is important for critical IoT applications) and b) the bottlenecks that are points where nodes are overloaded with traffic and need to be smoothed over to improve the QoS parameters (losses, flow, delay). It should be noted that load balancing, path length, and interference can be in conflict with each other. For instance, improving load balancing routing leads to an increase in the path length, as well as prevent interference can impair load balancing and increase path length. In addition, owing to the complex hop-to-hop nature of the communication in these wireless networks, the effectiveness of the routing algorithms have a great influence on the quality of the network.

This paper aims to propose an extension of the multiobjetive routing ILP model in [23], which was carried out in Wireless Sensor Networks (WSNs), to the routing optimization problem in WMNs towards IoT system, with focus on multimedia applications and sensing data from WSNs. The routing problem is based on three objectives: (i) minimize the path length (number of hops), (ii) minimize the bottleneck, and (iii) minimize the interference in the path.

The limited transmission speed provided by the IEEE 802.15.4 used in WSNs and the simple traffic application of WSN (i.e. single kind of application) did not enable to apply the existing model and algorithm in [23] for WMNs with mixed traffic, since the capacity and constraints characteristics of these wireless networks are very different. Therefore we present a reformulation of most objective functions in order to adequate to WMNs based on mixed traffic of future IoT as well as a novel multi-objective routing algorithm. As a result of this, we can take full advantage of WMN characteristics to achieve the overall network performance.

To the best of our knowledge, this is the first work in WMNs that takes into account these three conflicting objectives to achieve a more optimized selection of data routes for IoT applications based on mixed traffic. In addition, a comparative analysis is conducted between two multi-objective routing algorithms $[21,22]$ for multihop wireless networks against the proposed algorithm in this article that are based on ILP (Integer Linear Programming) mathematical models. In this way it can enable us to analyze important aspects of multi-hop wireless communications in greater depth.

The algorithms were simulated in a multi-channel network scenario. Mixed traffic models were used to represent current IoT applications with several requirements, such as video, audio, and sensing data. Moreover this article also provides the first study of multi-objective routing optimization algorithms based on ILP for multi-hop wireless networks. Furthermore, it should be emphasized that none of the approaches were previously assessed in a scenario with external interference, and thus this study can make a valuable contribution to research by carrying this out here.

This article is structured as follows: In Section 2, there is an analysis of the most relevant work on multi-objective routing approaches for multi-hop wireless 
networks. In Section 3, there will be a description of model multi-objective and present the algorithm MAXI (Multi-objective routing Aware of miXed traffIc for lowcost wireless backhauls) with main features of approach. In Section 4, we set out the simulation results in NS3. The conclusion and suggestions for future work are summarized in Section 5.

\section{Related Works}

The described studies in this section focus on works that offer solutions that involve the use of multiple objectives to determine the routing.

Boushaba, Hafid, and Gendreau [24] proposed a sourcebased routing approach for WMNs, which aims to improve network efficiency by introducing a new metric to determine the link quality, by combining the packet loss rate with interference caused by the inter-flow and intraflow. This approach seeks to maintain the balance of the gateway by selecting the interface that receives flows on the basis of the average queue size. When two metrics are combined, routes of a good standard can be selected. However, the problem of the bottleneck is critical in the neighborhood of the gateway, when it is not addressed at the intermediate links of the path. Links that are unable to directly bind to the gateway cause secondary bottleneck points on links with good quality, resulting in packet retransmission and packet loss rate because the device has a full queue.

The study with the title Quality of Experience-based Routing in Multi-Service Wireless Mesh Networks [25] employs a QoE-based method of unsupervised reinforced learning to determine the routing in WMNs, and seeks a better experience for the user. The network flows are divided into three types of services (video, audio, and data transfer), and there is a knowledge database of QoS constraints (delay, packet lost rate, etc.) for each service to determine the routes. At each time interval, this information is disseminated so that the nodes can update their QoE values through objective calculations. The approach determines individual routes for each type of service without taking account of the load balancing in the network.

Zimian Hao and Yingxia Li propose a routing approach for multi-mesh gateways [26] which includes an adaptation of the AODV protocol to disseminate load-aware metrics across the network. The RREQ messages from AODV aims to update the route tables with information about the traffic load on each channel. The RREP messages are used to update the tables with the paths when the destination is found, by determining which links will be used on the route. Although this type of approach seeks to optimize the transmission medium, it creates an overload of control packets that will reduce the efficiency of the network, as well as controlling the path length needed to reach a certain gateway. In this way, it can both increase the packet delay and the number of packets delivered, as it adds to contention and bottleneck in the network.

Alwan and Nuha [27] conducted an analysis and employed a multi-objective optimization method to perform the WMN routing using Dijkstra's algorithm and the weighted sum of the objectives (delay and link capacity) to determine the WMN routes. The demonstrated method only applies to objectives with linear behavior, and restricts the use of other metrics to determine the routes. The results obtained should be investigated in more realistic environments for a better analysis of the objectives; for example the delay in the delivery of packets might be caused by several situations such as interference, path length, and fairness.

A mathematical formulation for the multi-objective routing problem in hybrid WMN for smart city scenarios is proposed in [28], where there is a wide range of devices such as routers, mobile phones, sensors, notebooks, etc. The approach sets out four main factors that must be optimized: path length, delay, packet loss, and energy consumption. In general, energy consumption is not a crucial concern in WMN, since in most cases, this kind of network does not have serious energy constraints. The mathematical model is solved through an evolutionary approach, this involves the Strength Pareto Evolutionary Algorithm (SPEA) in a distributed way, since each node is able to calculate its own route (source node) to any other node (target node) by following the mathematical model. All the tests were carried out without the use of a simulator or testbed to validate the definitions specified by the approach. However, this approach is based on some unrealistic assumptions such as the fact that delay is statically defined for each link. Besides, delay and path length are overlapped objectives, since the path length influences significantly the end-to-end delay. In addition, the packet loss rate in a link is determined by means of the distance between the devices that make up the link that is designed to establish the signal strength in the receiver node, which does not depict precisely the link quality. Despite the delay and packet loss are results of regions of load bottlenecks, it is interesting to include an objective function based on load balancing in order to minimize the bottlenecks and thus it can avoid the delay and packet loss before they happen.

Gálvez and Ruiz proposed the routing algorithm called Joint Routing, Channel Assignment and Rate allocation Heuristic (JRCAR) in [21]. JRCAR determines the routing for the flows to reduce the overload links, while it seeks to select shorter paths that reduce the path length of each flow. The authors designed a mathematical model of integer linear programming that represents the routing problem in which the network bottleneck is used as an objective function and the number of hops performed by the paths is modeled as a constraining factor. 
This approach selects paths that reduce the network bottleneck (load balancing) to enable the algorithm to distribute the flows in a larger number of links. JRCAR defines the bottleneck of any path in WMN as the highest communication link usage, where the term "usage" is defined as the number of routes that are included in the link. The aim of this study is to balance the instantaneous traffic in the network through the distribution of the flows, by optimizing the allocation of the channel links to achieve equitable loads in the network. This approach does not take account of the quality of the links and all the tests were carried out with a single type of simple and not very realistic traffic (i.e. only TCP application).

JRCAR generates the routes in the network by means of a pre-established order of flows. The path for each flow is calculated through the number of candidate paths in ascending order. In other words, the routes of the nodes which have fewest alternatives are calculated first. As a result, JRCAR can keep control of its path length, since the most feasible paths have a greater chance of obtaining a path with a similar/equal number of hops while it does not increase the number of bottlenecks.

Mello et al. [22] present a heuristic called Joint approach for Improving Load balancing and Path length (JILP) for a bi-objective optimization of WMN, where the routes with shortest paths are selected. JILP is responsible for finding solutions for a integer linear multi-objective programming model that can reduce the number of hops and bottlenecks of the routes in a WMN. This model is able to maintain the lowest bottlenecks in the network, and keep control of the routing stability to avoid any unnecessary change of routes. The routing approach is then combined with a channel assignment algorithm to improve network efficiency. However, this approach does not take into account that the scenario is influenced by natural physical phenomena, such as interference and signal fading. This may not be enough to provide a good performance, largely because it is subject to signal quality degradation in some regions of the network.
The JILP algorithm is used to add or remove new routes when the flows of the network begin or finish, respectively. If adding a new route leads to an increase in the bottleneck, JILP recalculates the routes involved in the affected links. In a similar way to the JRCAR approach, JILP controls the maximum length of the paths, by means of the same stretch factor as JRCAR, to avoid any degradation of the network performance, although it selects short paths when these include only the flows that pass through the bottleneck. Hence, JILP provides a local routing solution based on traffic demand, which reduces the routing overhead and route fluctuations. However, it can prioritize the load balancing routing rather than the path length, since it seeks to keep control of the local bottleneck when adding or removing the flow. In addition, it only attempts to reduce the path length for the flows that pass through the bottleneck if it is impossible to obtain the previous smaller value of the bottleneck.

\subsection{Comparative Analysis of Related Works}

Table 1 provides a comparison between different routing approaches of WMNs. This table shows that in the scenarios of both WMNs there have been few papers that set out a mathematical model to represent the problem of routing in wireless networks and that use two or more objectives to determine the routes. It is important to stress out that none of these approaches addresses the requirements of each application of the mixed traffic when calculating the routes. There are two similar approaches among those included, JRCAR [21] and JILP [22], that stand out being the most efficient. This is because they are based on the ILP mathematical model and take into account the main objectives that either directly or indirectly influence the performance parameters (delay, throughput, packet loss rate). However, the used scenarios to evaluate these approaches lack features for current and future applications (such as traffic with different QoS requirements and transmission data rate) and network scenario (such as, a physical layer model that reproduces different levels of interference).

Table 1 Related work comparison

\begin{tabular}{|c|c|c|c|c|c|c|c|}
\hline Related Work & Math. Model & Path Length & Link Quality & Load Balancing & Energy & Traffic & Type \\
\hline SBR [24] & no & no & yes & yes & no & simple & centralized \\
\hline QoEbRS [25] & no & yes & yes & no & no & mixed & centralized \\
\hline LAM [26] & no & no & no & yes & no & simple & distributed \\
\hline DijkstraW. [27] & no & yes & yes & no & no & simple & centralized \\
\hline RSCSPEA [28] & yes & yes & no & no & yes & simple & distributed \\
\hline JRCAR [21] & yes & yes & no & yes & no & simple & centralized \\
\hline JILP [22] & yes & yes & no & yes & no & mixed & centralized \\
\hline MAXI & yes & yes & yes & yes & no & mixed & centralized \\
\hline
\end{tabular}




\section{MAXI - Multi-objective routing Aware of miXed trafflc for Low-cost Wireless Backhauls}

In this section, we describe the mathematical multiobjective optimization model of ILP (Integer Linear Programming) and an algorithm called MAXI - Multiobjective routing Aware of miXed traffIc to address the single-path routing problem in WMNs. The MAXI algorithm employs techniques to simplify the complex task of selecting the set of Pareto optimal solutions.

\subsection{Integer Linear Programming Model}

The proposed multi-objective optimization model for the routing in the WMNs is based on three objectives: reducing the number of hops, reducing the network bottleneck links, and avoiding the use of low quality links, which significantly affects the application traffic performance of WMNs. These objectives conflict with each other because there is no best solution to achieve all three objectives to an optimal degree. First of all, the number of hops in a required path must be minimized in order to reduce the end-to-end delay and contention. Figure 1 shows the proposed the ILP optimization model.

The interference and contention caused by external and internal sources have a serious impact on the WMN traffic performance. However, the physical layer described by the IEEE 802.11 standards can enable the metrics to measure the quality of transmission of a wireless link in a more precise way [29].

One key feature of the future IoT applications is the provision of communication services for the most diverse types of applications, which can allow the transmission and reception of mixed traffic. WMNs must offer varied levels of quality of service (QoS) for different types of traffic (data, voice, video), which requires a greater transmission capacity. To achieve this, the objective function of load balancing must take into account the different features of each type of traffic, because applications (e.g. video streaming) with higher transmission rates consume more bandwidth resources in WMNs and for this reason cannot be equated with applications (sensor data) that have considerably lower transmission rates.

It is possible to assign channels between different radios in WMNs that may exist on the devices, and thus improve the data traffic and reduce network contention. The use of multiple channels and multiple radios benefits load balancing between the wireless links since it relieves the total amount of data that make use of the spectrum. Each link has a maximum resource capacity that can be used by the network before it starts causing bottleneck problems, for instance packet loss rate.

WMNs were modeled as a graph $G=(V, E)$, where $V$ is the set of vertices that represents the WMN nodes, and $E$ is the set of communication links between two network devices. The link $e_{s d} \in E$ between the nodes $s, d \in V$ can only exist if the device $s$ accomplishes a data transmission to the node $d$. Every node $s \in V-\{i\}$, where $i$ is the sink, can be responsible for originating one or more data streams called $f_{s} \in F$, where $F$ is defined as the set of all the flows generated in the network. All the flows of the WMNs have the Internet node (sink node $i$ ) as their destination node. The Internet node is responsible for establishing Internet connection with wired networks.

Following this, we will outline the proposed ILP model (shown in Fig. 1), and describe each objective function. Table 2 describes the list of variables used in the model.

Number of Hops. A linear programming model is used to find the shortest paths for $f$ flows in $F$; the purpose of the objective function (1) in the model is to find the minimum number of hops for the solution.

$$
\begin{aligned}
\text { Minimizee } & \left\{\sum_{e_{s d} \in E} a_{s d}\right\} \\
\text { Minimize: } & \left\{\sum_{e_{s d} \in E} l_{s d} \cdot a_{s d}\right\} \\
\text { Minimize: } & \left\{\left[\max \left(\left\{\sum_{d \in V} \sum_{\left\{f \in F_{s d} \mid c_{s d}=c\right\}} T^{f}\right\}, \forall c \in C_{s}\right)\right], \forall s \in V\right\} \\
\text { subject to: } & \sum_{d \in V} a_{v d}-\sum_{s \in V} a_{s v}=f_{v}, \forall v \in V, v \neq i \\
& \sum_{s \in V} a_{s i}=|F|, i=\sin k \\
& a_{s d} \geq 0
\end{aligned}
$$

Fig. 1 The proposed multi-objective optimization ILP model 
Table 2 Description of variables used in system model

\begin{tabular}{ll}
\hline Variable & Description \\
\hline $\boldsymbol{V}$ & Set of vertices. \\
$\boldsymbol{E}$ & Set of edges (links communication). \\
$\boldsymbol{e}_{\boldsymbol{s} \boldsymbol{d}}$ & Specific edge $e_{s d} \in E$ for vertices $s, d \in V$. \\
$\boldsymbol{a}_{\boldsymbol{s} \boldsymbol{d}}$ & Sum of flows using the edge $e_{s d .}$ \\
$\boldsymbol{l}_{\boldsymbol{s} \boldsymbol{d}}$ & Variable representing the quality of the link $e_{s d .}$ \\
$\boldsymbol{f}_{\boldsymbol{v}}$ & Total of flows sourced in $v$, by $v \in V$. \\
$\boldsymbol{F}_{\boldsymbol{s} \boldsymbol{d}}$ & Set of flows using the link $e_{s d .}$ \\
$\boldsymbol{F}$ & Set of all flows. $\left(\sum_{v \in V} f_{v}=|F|\right)$ \\
$\boldsymbol{T}^{\boldsymbol{f}}$ & Flow rate $f \in F$. \\
$\boldsymbol{C}_{\boldsymbol{s}}$ & Channels assigned to adjacent links of node $s \in V$. \\
$\boldsymbol{c}_{\boldsymbol{s} \boldsymbol{d}}$ & Channel of link $e_{s d} \in E$. \\
\hline
\end{tabular}

Wireless Links with Low Quality The objective function (2) uses the $l_{s d}$ variable to minimize the use of low-quality links for the paths.

A transmission originating from a node to any other neighbor node is only successful if the Signal to Interference plus Noise Ratio (SINR) for the receiving node is above a certain threshold. The value of SINR is used as a basis for obtaining the $I R$ (Interference Ratio) quality metric $[24,30]$ in which the values are represented in a range from 0 to 1 . The $I R$ is obtained by calculating the relation between the value of the SINR and the SNR (maximum tolerable interference level) for the node receptor. The $I R$ will be used as a quality metric for the WMNs since it is a more accurate alternative for link quality measurements in wireless networks. The variation of $I R$ represents an interval from 0 to 1 in which the links can be classified in terms of their quality. Equation (7) normalizes the $I R$ values so that the link can be classified into low, intermediary and high quality categories.

The variable $l_{s d}$ is responsible for storing the normalized value of $I R$, represented by the $I R_{s d}$ variable, which will indicate the quality of the link between the source node $(s)$ and the destination node $(d)$ as expressed in Eq. (7). The $l_{s d}$ variable is used by the objective function that reduces the number of low quality links (Eq. 2) and must have a value equal to zero (as defined in Eq. (7-a) so that it can represent links with a high quality. This is because these links do not add value at the cost of the objective function if the link is used. However, when a link has low or medium quality (as defined in Eqs. (7-b) and (7-c)) the value of $l_{s d}$ must be greater than zero and lower than, or equal to, 1 (which represents a link with very poor quality). Hence, IR was normalized so that the link quality is classified in a range from 0 (low quality) to 1 (high quality) and moreover, objective function (2) seeks to minimize the utilization of links for the path with high values of $l_{s d}$ (one).
An empirical study was carried out to estimate these three link classifications through a set of simulations, i.e. five topologies and six random seeds were generated for each configuration of the number of nodes $(10,20,30,40$, 50 nodes), resulting in a total of 30 simulations for each set of node. Table 3 shows the main configuration parameters of scenario of the empirical study, however all the parameters can be seen in Table 6 .

$$
l_{s d}=\left\{\begin{array}{lll}
0, & I R_{s d} \geq T_{h} & (a) \\
1-\left(\frac{I R_{s d}-T_{l}}{T_{h}-T_{l}}\right), & \left(T_{h}>I R_{s d}>T_{l}\right) & \text { (b) } \\
1, & I R_{s d} \leq T_{l} & \text { (c) }
\end{array}\right.
$$

The $T_{l}$ and $T_{h}$ thresholds were fixed so that the the link quality could be divided in accordance with the $I R$ value that is obtained. Figure 2 shows the histogram of number of packet transmissions with failures and successes related to the $T_{l}$ and $T_{h}$ thresholds, respectively, as well as the value of $I R$. It is important to note that the packets of this histogram are only derived from the physical layer taking into account the thresholds of SINR and SNR.

The $T_{l}$ threshold was set at 0.60 , as there were very few successful transmissions when the IR was lower than this value; for example, there were $40.43 \%$ failed transmissions and $0.08 \%$ successful transmissions, so the chances of success are low. The $T_{h}$ threshold was set at 0.75 , since there were very few failures in the transmissions when $I R$ was higher than, or equal, to $0.75 ; 15.27 \%$ of the transmissions failed and $39.86 \%$ of the transmissions were carried out successfully, so the chances of success were high. In light of this, the $T_{l}$ and $T_{h}$ threshold values were set at 0.60 and 0.75 , respectively.

In Fig. 2, 44.3\% of failures and 59.34\% of successes occur when the value of the IR lies between the limits of $T_{l}$ and $T_{h}$, i.e. there is little difference between success and failure when links with levels of intermediary quality are used. It is also worth noting that a number of representative success and failure packets are found in this interval. Equation (7b) assigns the value of the $l_{s d}$ variable in this interval close to 0 when the IR value approximates to the value of $T_{h}$, which is the region where the highest number of successful transmission occurs, and close to 1 when the IR approximates to the value of $T_{l}$, a region where

Table 3 Empirical study: NS-3 simulation parameters

\begin{tabular}{ll}
\hline Transmission Power & $22(\mathrm{dBm})$ \\
Power Detection Threshold & $-78.0(\mathrm{dBm})$ \\
Carrier Detection Threshold & $-62.0(\mathrm{dBm})$ \\
Channel Width & $20 \mathrm{MHz}$ \\
Propagation Loss Model & Log Distance \\
Physical layer (NS-3) & $802.11 \mathrm{n}$ (SpectrumWifiPhy) \\
Topologies Area & $400 \mathrm{~m} \times 400 \mathrm{~m}$
\end{tabular}




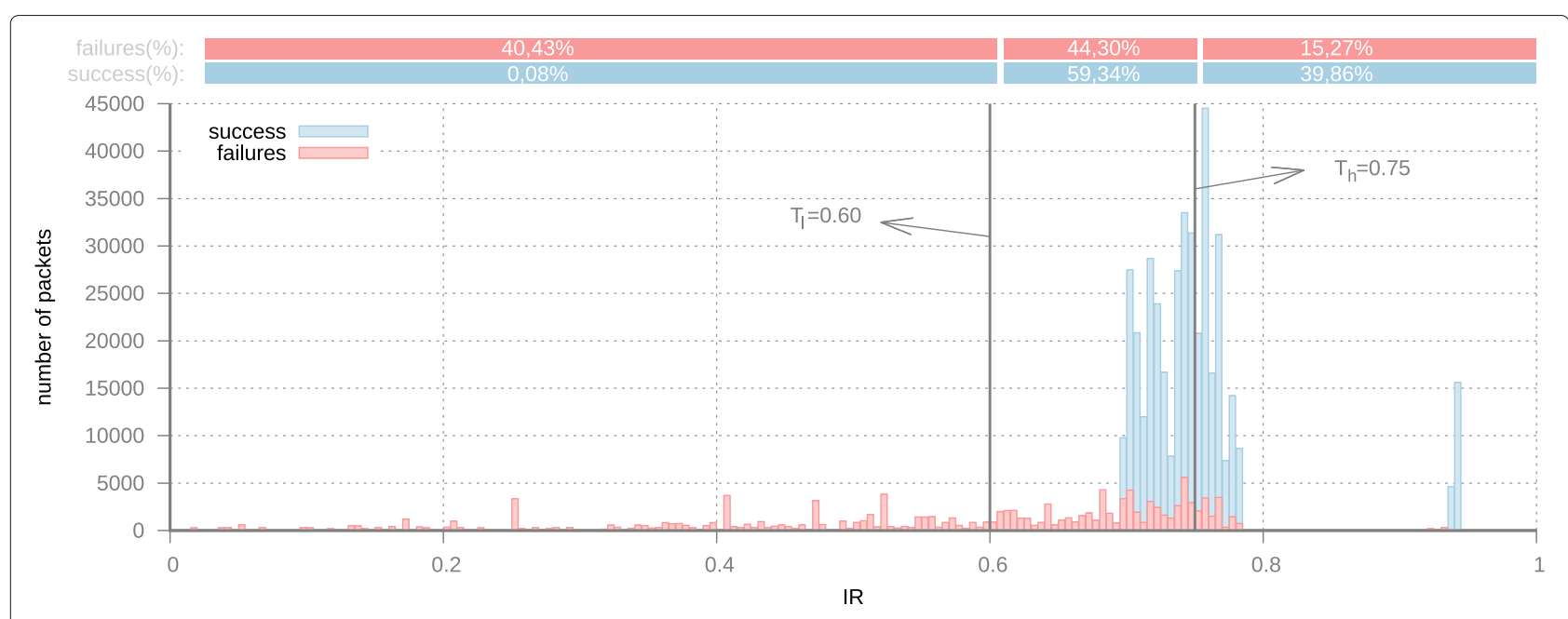

Fig. 2 Histogram of successes and failures in transmission as estimated by the IR (link quality metric)

there are no or few attempts that result in a successful transmission.

Network Bottleneck On the one hand, the distribution of flows in a non-balanced way increases the number of agglomerated flows at the same node (i.e. cause a bottleneck). This increases the number of forwarded packets and hence leads to delay and adds to the packet loss rate. However, it is possible to employ a set of non-overlapping channels between the different radios presents in the mesh routers through a channel assignment algorithm so that the contention can be mitigated and there can be an improvement in the data traffic performance. Hence, objective function (3) minimizes the network bottleneck through a reduction of the largest amount of flow data used by a single channel for all the nodes of network.

Constraints The model imposes three constraints that can assist in providing a valid solution, i.e. a route in the network topology. The constraint (4) ensures that every node (vertex) can only generate a specific number $f_{v}$ of flows and the constraint (5) ensures that the Internet Gateway (i.e. the Internet node) is only able to receive the number of flows generated in the network. This combination of restrictions (4) and (5) ensures that all the flows have a path to the Internet node. The constraint (6) provides non-negative values for the number of flows on the links in $a_{s d}$ (The variable $a_{s d}$ is defined as the sum of the flows that use the edge $e_{s d}$ on their route). Although constraints (4), (5), and (6) do not guarantee that there will not be any loops, the constraint (6) ensures that if there is a viable solution for creating loops, this will increase the cost of the objective function. As a result, this solution will not be an optimal for minimization problem on the basis of the optimality principle of Dijkstra routing algorithm [31].

\subsection{Multi-objective routing Aware of miXed trafflc for low-cost wireless backhauls (MAXI)}

The Multi-objective routing Aware of miXed traffIc (MAXI) is a multi-objective algorithm for solving the routing problem that was modeled in the previous sub-section. The MAXI basically uses an algorithm to determine paths at a lower cost (e.g by using Dijkstra's algorithm) in a graph that represents a WMN. The values related to the objective functions, i.e the values associated with edges, are changed depending on which nodes have already been assigned their flow routes.

The MAXI algorithm combines three weighted objective functions (path length minimization (1), minimization of links with low quality (2) and mitigation of network bottlenecks (3)) at each iteration, to calculate a route for the new flow. Algorithm 1 employs the weighted sum method to assign the weights for each objective function to meet the requirements of the specific application of that flow in line 4. After this, a minimum cost path is created using the MCPathfunction (sender; receiver; GRAPH) for the $f^{s}$ flow (line 5), and the path is incorporated in the solution set that will contain all the routes for every flow (as can be observed in line 6).

The procedure update_values_edges $\left(w_{p}, w_{l}, w_{b}\right)$ defined in lines 10 to 20, calculates the single objective function by combining the three objectives shown in subsection 3.1, where the $w_{p}, w_{l}, w_{b}$ variables are weighted path length, links with low quality, and network bottlenecks, respectively. To achieve this, it is necessary to normalize the values at the same interval. The edge values for link quality and the number of hops are already in same interval $[0,1]$, but the edge values for network bottleneck are not. The sum of all the flow rates in adjacent 


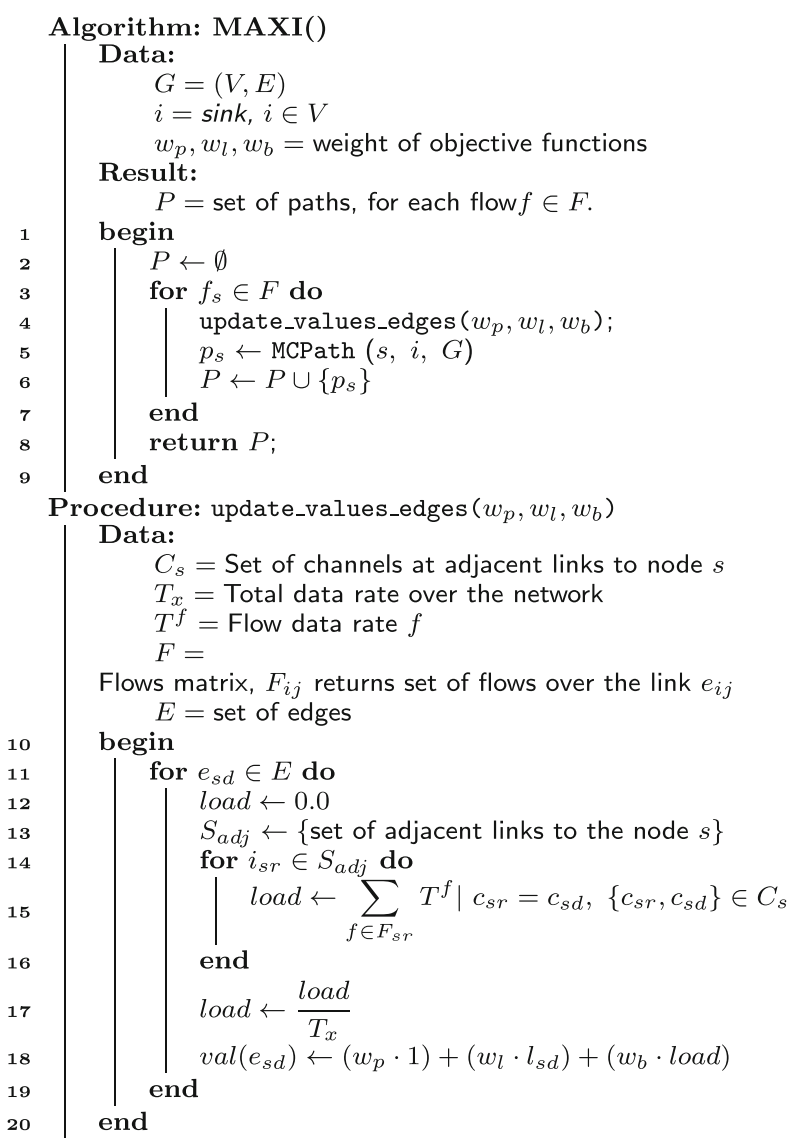

Algorithm 1: MAXI - Multi-objective routing Aware of miXed TraffIc

channels for a set of nodes that use the same channel are calculated in line 15. After this sum has been calculated for the value of the load variable, it is divided by the maximum load of the network so that it can be standardized in the interval $[0,1]$. Finally, the values of the objectives are combined in the single objective function by using the weights assigned in line 18 of the MAXI Algorithm.

A route is generated for a given flow type at every iteration algorithm. The Table 4 shows the stages for calculating the routes represented by Fig. 3. Assuming that the opening sequence of the flows in mesh routers is given by $F=\{D, C, A, E, B\}$ and that each mesh router will generate two types of flows, i.e. $f^{1}$ and $f^{2}$. The application that generates $f^{1}$ has a rate of $256 \mathrm{kbps}$ and its assigned weights are $w_{l}=0.6, w_{p}=0.2$ and $w_{b}=0.2$. In contrast, the application that generates $f^{2}$ has a rate of $1024 \mathrm{kbps}$ and its assigned weights are as follows: $w_{l}=0.2, w_{p}=$ 0.2 and $w_{b}=0.6$. Given the $F$ order of the devices, priority is given to every node so that it can generate the routes between the flows originating in that node, as is shown in the example given in Table 4, where initially, the route for every node is generated for the flow of $256 \mathrm{kbps}$ and soon afterwards for the flow with a transmission rate equal to 1024 kbps.

For each sequence, the Table 4 shows the data load on each edge that is used by one or more flows. After adding up all the loads of every flow by means of a specific link, the values are incremented as soon as the new routes have been calculated. The cost variable displays the value of the path selected for the $f_{s}^{x}$ stream, where $s$ is the source node and $x$ the type of flow.

The ninth and tenth stages show the difference between the routing for each flow type: the $f_{b}^{1}$ flow prefers paths that have better quality indicators, since they have a greater weight for the quality objective function of the links $\left(w_{l}\right)$. However, a path that distributes the data load in a balanced way over the network, has been selected for the $f_{b}^{2}$ flow. Thus, distinct paths are selected for each flow, even though they have the same source node (i.e. the $B$ node).

Table 4 MAXI Execution sequence

\begin{tabular}{|c|c|c|c|}
\hline N & Flow (Rate) & Selected path & Load data on the edges \\
\hline 1 & $f_{d}^{1}(256 k b p s)$ & (D) $\rightarrow$ (C) $\rightarrow$ (S) cost $=0.70$ & (D) $\rightarrow$ (C) $=256 \mathrm{kbps}(\mathrm{C}) \rightarrow$ (S) $=256 \mathrm{kbps}$ \\
\hline 2 & $f_{d}^{2}(1024 \mathrm{kbps})$ & (D) $\rightarrow$ (C) $\rightarrow$ (S) cost $=0.548$ & $(D) \rightarrow(C)=1280 \mathrm{kbps}(\mathrm{C}) \rightarrow$ (S) $=1280 \mathrm{kbps}$ \\
\hline 3 & $f_{c}^{1}(256 \mathrm{kbps})$ & (C) $\rightarrow$ (s) cost $=0.50$ & (D) $\rightarrow$ (C) $=1280 \mathrm{kbps}(\mathrm{C} \rightarrow$ (S) $=1536 \mathrm{kbps}$ \\
\hline 4 & $f_{c}^{2}(1024 \mathrm{kbps})$ & (C) $\rightarrow$ (s) cost $=0.404$ & $($ (D) $\rightarrow$ (C) $=1280 \mathrm{kbps}(C \rightarrow$ (S) $=2560 \mathrm{kbps}$ \\
\hline 5 & $f_{a}^{1}(256 k b p s)$ & $(A) \rightarrow$ (C) $\rightarrow$ (S) cost $=0.66$ & (A) $\rightarrow$ (C) $=256 \mathrm{kbps}(\mathrm{D}) \rightarrow$ (C) $=1280 \mathrm{kbps}(\mathrm{C} \rightarrow$ (S) $=2816 \mathrm{kbps}$ \\
\hline 6 & $f_{a}^{2}(1024 k b p s)$ & (A) $\rightarrow$ (C) $\rightarrow$ (S) cost $=0.676$ & $(A) \rightarrow(C)=1280 \mathrm{kbps}(\mathrm{D}) \rightarrow(C)=1280 \mathrm{kbps}(\mathrm{C} \rightarrow$ (S) $=3840 \mathrm{kbps}$ \\
\hline 7 & $f_{e}^{1}(256 k b p s)$ & (E) $\rightarrow$ (S) cost $=0.38$ & $\begin{array}{l}\text { (E) } \rightarrow \text { (S) }=256 \mathrm{kbps}(A) \rightarrow \text { (C) }=1280 \mathrm{kbps}(\mathrm{D}) \rightarrow \text { (C) }=1280 \mathrm{kbps} \\
(\mathrm{C} \rightarrow \text { (S) }=3840 \mathrm{kbps}\end{array}$ \\
\hline 8 & $f_{e}^{2}(1024 k b p s)$ & (E) $\rightarrow$ (S) cost $=0.284$ & $\begin{array}{l}\text { (E) } \rightarrow \text { (S) }=1280 \mathrm{kbps}(A) \rightarrow(C)=1280 \mathrm{kbps}(\mathrm{D}) \rightarrow \text { (C) }=1280 \mathrm{kbps} \\
(\mathrm{C}) \rightarrow \text { (S) }=3840 \mathrm{kbps}\end{array}$ \\
\hline 9 & $f_{b}^{1}(256 k b p s)$ & $(B) \rightarrow(A) \rightarrow$ (C) $\rightarrow$ (S) cost $=1.208$ & $\begin{array}{l}\text { (B) } \rightarrow \text { (A) }=256 \mathrm{kbps}(\text { (E) } \rightarrow \text { (S) }=1280 \mathrm{kbps}(A) \rightarrow(C)=1536 \mathrm{kbps} \\
(\text { D) } \rightarrow \text { (C) }=1280 \mathrm{kbps}(\text { C) } \rightarrow \text { (S) }=4096 \mathrm{kbps}\end{array}$ \\
\hline 10 & $f_{b}^{2}(1024 k b p s)$ & (B) $\rightarrow$ (A) $\rightarrow$ (E) $\rightarrow$ (S) cost $=0.944$ & 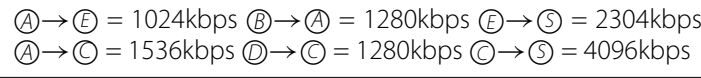 \\
\hline
\end{tabular}




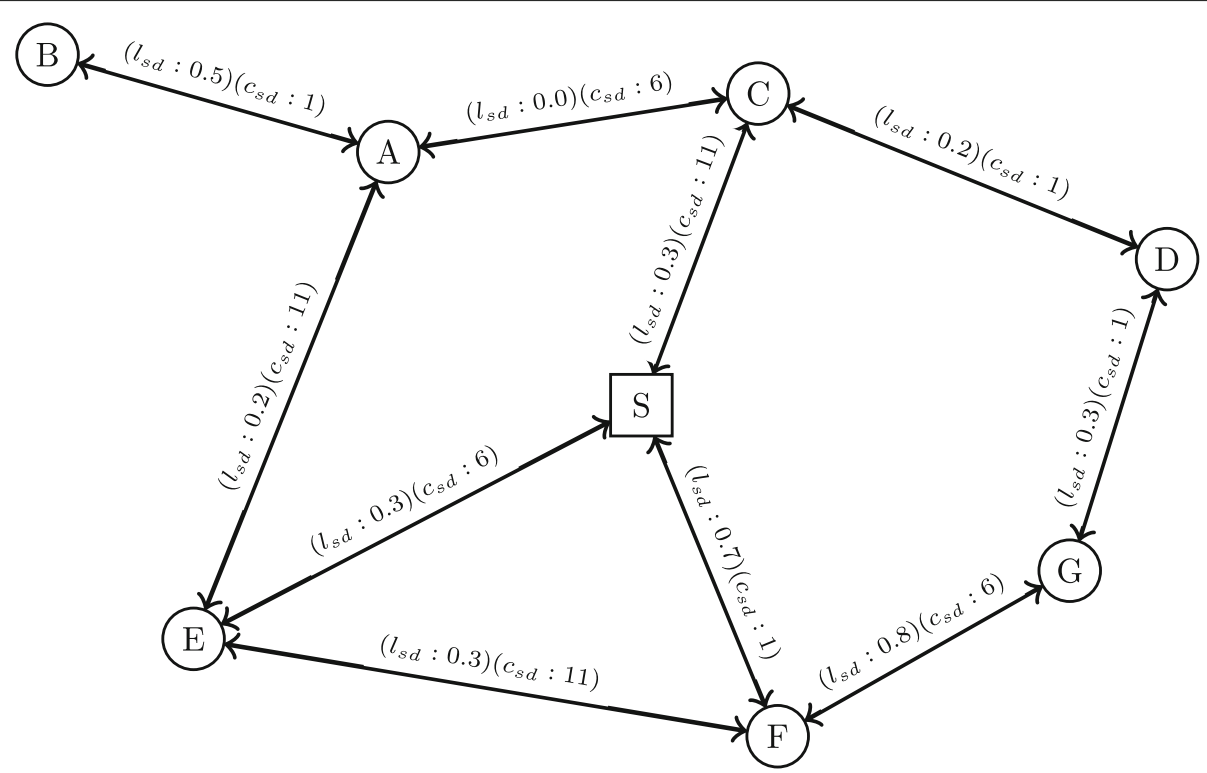

Fig. 3 Example topology of an WMN

\section{Simulation Results}

This section presents the comparison of the most relevant multi-objective routing algorithms based on ILP model JRCAR, JILP, and MAXI (proposed in the previous section) in the context of future IoT applications based on mixed traffic through a simulation study. The WMNs employed in the performance evaluation should provides key factors to enable an analysis to be conducted of the algorithms:

- The possibility of traffic with high capacity, to support a wide range of applications that may exist in an future IoT context.

- The risk of internal interference, caused by the network nodes themselves, and external interference, caused by devices that are not a part of the network.

For these reasons, we chose an elderly monitoring system as a suitable example of a set of IoT applications and the NS-3 network simulator to model WMNs that can support the performance requirements of this kind of system as well as to design different levels/types of interference for the wireless network and the applications running on it. This section is structured as follows: there is an outline of the IoT applications and wireless network scenario that is employed in Sub-sections 4.1 and 4.2 , respectively. The simulation results are discussed in Sub-section 4.3.

\subsection{Description of the loT Applications}

WMNs provide a multi-hop wireless infrastructure that enables high-bandwidth traffic to support a wide range of applications, and allows it to be used in several areas such as scenarios that require higher bandwidth (multimedia applications) or have variable requirements for each application (for instance, sensors). It should be noted that future IoT applications will combine several applications with different requirements [32,33]. Owing to the inherent features of future IoT applications, we believe this is a useful application scenario to compare the algorithms studied, since it provides a large number of applications and constraints that can be applied to the infrastructure of a LWB like WMNs.

One example of a future IoT application is the healthcare information system where advances in the portable wireless monitoring system [34] make it more comfortable, for example, for an elderly person to be cared for at a nursing home where he/she can be monitored by a portable system. In light of these healthcare applications, there are specific monitoring systems for particular health problems or preventive monitoring [32,33]. The cost of health services and public welfare has been increasing in recent years, especially in countries where people have a high life expectancy. Thus, the challenges to obtain quality services, which meet the needs of elderly people for example, require these monitoring systems to evolve [35].

The large number of portable devices and sensors (accelerometers, gyroscopes, intelligent clothing, actuators, etc.), used in monitoring systems of the elderly, has led to a very wide range of applications. In addition, wearable sensors have the ability to monitor a patient's vital signs: electromyogram (EMG), electroencephalogram (EEG), respiration rate, sound of heart beats, blood glucose level, oxygen saturation, and body temperature. 
Finally, it is possible to collect information about mobility or falls [32, 34-38].

The monitoring devices can be grouped according to their purpose, location, and monitoring systems [36], for example:

- Devices for individual use as an accessory (watches, bracelets, necklaces, bandanas).

- Implantable devices (miniaturized video cameras, gastric pressure measurement, continuous glucose monitoring).

- Devices or multimedia systems (video cameras, depth sensor cameras, microphones, smartphones).

- Devices incorporated into clothing.

- Devices adapted to objects, furniture or the floor of the house.

All devices present in a monitoring system for the elderly should have a mean of communicating with the health monitoring center, which manages the care services and makes it possible to route the data collected by the sensors. Each application, which is built on a set of devices, has different specifications such as data rate and latency $[34,39]$. Thus, the wireless backhaul of the WMN is a very important to support mixed traffic originating from more than one application using the network to forward the data to a given environment.

In general, the communication architecture for health services can be divided into three categories. The first consists of a network of sensors and devices that is directly linked to the monitored individual. The second level is the WMN which is responsible for sending information from the sensors/devices to the Internet. The third level is the wired network that forward the gathered data by the WMN to the remote monitoring center. Usually, the third level is not necessary if there is a local monitoring center, i.e. it is in the same place that the health services is offered. Thus, all the WSN traffic or that from a specific device (video, microphone, smartphone) is passed through the mesh routers to the medical staff for local or remote monitoring center $[35,40]$. We assume a local monitoring center in this article. The healthcare information system has several critical requirements that must be taken into account, such as reliable data delivery, privacy, system response time, etc. [40]. The communication network must provide support to meet these requirements, this means that the links must have enough transfer capacity to ensure the generated information can be transmitted and the data is sent using links with good quality to ensure better efficiency.

The healthcare system involves a set of devices, each one with specific purpose, constraints, and responsible for generating a flow in the network. In this article, an elderly monitoring is made up of two applications: a fall monitoring application, generating multimedia flow classes, and a vital sign monitoring application, generating sensor data flow classes [32, 33, 36]. Each application flow has restrictions for these parameters: the fall monitoring application uses multimedia traffic and has inherent restrictions of the loss rate, being desirable no more than $20 \%$ to $30 \%$ losses during data transmission. However, the vital sign monitoring application has delayed packet delivery restrictions, and it is desirable a delay less than $125 \mathrm{~ms}$ $[34,38]$.

These two applications can share the same LWB infrastructure when sending their data. The fall monitoring application can use video devices such as an RGB camera, which has a data rate of approximately $264 \mathrm{kbps}$, and a depth sensor [32], which generates a flow at an approximate rate of $1 \mathrm{Mbps}$. The vital signs monitoring application uses a set of sensors that perform measurements of a patient's signals. This set of sensors may vary in accordance with the type of monitoring that needs to be carried out; however, most of them are connected to the patient's body $[34,35]$. In this article, the vital signs monitoring application employs three types of sensors (each one being responsible for generating a type of flow in the network): electrocardiogram (ECG), with a data rate of $3 \mathrm{kbps}$, oxygen saturation (SpO2), with a rate of $32 \mathrm{kbps}$, and blood pressure sensor, with a rate of $10 \mathrm{bps}[34,38]$. Table 5 summarizes the applications used to perform the tests and the types of flows that each device generates.

The choice of weights are based on the set of requirements of the QoS parameters on each application as well as the combination of them in the elderly monitoring system, i.e. loss rate between $20 \%$ and $30 \%$ for fall monitoring application and delay less than $125 \mathrm{~ms}$ for vital sign monitoring application [34, 38]. In other words, the requirements and objective functions are associated by giving more and less weight to the objective functions that is somehow influenced and related to the set of application requirements as well as we set distinct weights for the same objective functions in different applications so that these applications can coexist in the network by taking advantage of the set of alternative routes.

The weights used in the MAXI approach during the simulations are: $w_{p}=0.50, w_{l}=0.20, w_{b}=0.30$ for vital

Table 5 Summary of the monitoring system for the elderly used in the simulations

\begin{tabular}{lll}
\hline Application & Devices & Rate \\
\hline Fall monitoring & RGB Camera & $264 \mathrm{kbps}$ \\
& Depth Sensor & $1024 \mathrm{kbps}$ \\
Vital Signs Monitoring & Electrocardiogram (ECG) & $3 \mathrm{kbps}$ \\
& Oxygen Saturation (SpO2) & $32 \mathrm{kbps}$ \\
& Blood Pressure & $10 \mathrm{bps}$ \\
\hline
\end{tabular}


signs monitoring and $w_{p}=0.35, w_{l}=0.45$ and $w_{b}=0.15$ for fall monitoring. As the application for vital signs monitoring have strong end-to-end delay constraints of the packets, it was decided to give more weights to the $w_{p}$ which is referring to the path length. MAXI uses a median value for the load balancing weight $w_{b}=0.30$ in order to reduce the delay as this helps to reduce waiting time in the queue.

The weights for the fall monitoring application aims to achieve low packet loss rates, so higher values are assigned for the quality goals of the links and path length. The MAXI approach has the weight for the set of links with low quality $\left(w_{l}=0.45\right)$ and the weight for path length $\left(w_{p}=0.35\right)$ high, so the selected paths tend to have few hops and few links with low quality. The fall monitoring has preference in the order of selection of the routes.

\subsection{Network Scenario}

The NS-3 simulator was used in the tests for a better analysis of each algorithm's performance. NS-3 enables the simulation of a WMN by gathering a more complete set of the network features. Table 6 shows the general parameters used in the simulation.

Physical layer model. Physical layer models are mainly responsible for modeling the reception and transmission of packets and recording energy consumption. The NS3 simulator provides by default two modules to represent the physical layer of devices for wireless networks: YansWifiPhy [41] and SpectrumWifiPhy [42].

Table 6 NS-3 simulation parameters

\begin{tabular}{ll}
\hline Transmission Power & $22(\mathrm{dBm})$ \\
Power Detection Threshold & $-78.0(\mathrm{dBm})$ \\
Carrier Detection Threshold & $-62.0(\mathrm{dBm})$ \\
Channel Width & $20 \mathrm{MHz}$ \\
Propagation Loss Model & Log Distance \\
Physical Layer & IEEE $802.11 \mathrm{n}$ \\
Physical layer model (NS-3) & SpectrumWifiPhy \\
Transmission Rate & $80 \mathrm{Mbps}$ \\
Frequency Band & $5 \mathrm{GHz}$ \\
RTS/CTS & Disabled \\
MAC layer & Adhoc - CSMA/CA \\
Maximum Retransmissions Number & 7 \\
Maximum Queue Time & $250 \mathrm{~ms}$ \\
Topologies Area & $400 \mathrm{~m} \times 400 \mathrm{~m}$ \\
Minimum Distance Among Nodes & $20 \mathrm{~m}$ \\
Maximum Distance Among Nodes & $40 \mathrm{~m}$ \\
Number of Radio Interfaces & 3 \\
Stretch Factor (JRCAR and JILP only) & 2.5 \\
\hline
\end{tabular}

The YansWifiPhy model is the standard adopted by NS-3 and is the most widely used model in studies and implementations, such as in JRCAR [21] and JILP [22], of Wi-Fi solutions using the NS-3 simulator. Nevertheless, the SpectrumWifiPhy model is an alternative implementation and allows scenarios to be created with the aid several technologies that coexist in the same channel, and thus cause interference among the channels in the same band. In addition, SpectrumWifiPhy provides tools capable of simulating external interference through the generation of noise [42]. For these reasons, we adopt the SpectrumWifiPhy model as physical layer model in the simulations.

In this study, the channel assignment algorithm proposed by Gálvez et al. [21] is used which relies on the distance (in hops) from the devices to the gateway and the quality of the links. This algorithm has been employed in related works of WMN $[21,22]$.

In this way, the simulations were performed in scenarios where the number of nodes ranged from 10 to 50 nodes (Table 6) that, on average, represents the number of devices in the monitoring applications for falls and vital signs. The configuration of traffic loading in the WMNs based on the percentage of mesh routers that actually generate flows, i.e. the percentage of active nodes, which is $100 \%$ of active nodes. Each mesh router generates two flows for every IoT device, i.e. 10 flows (two different applications, displayed in the Table 5). Ten topologies and four random seeds were generated for each configuration of the number of nodes, resulting in a total of 40 simulations for each set of nodes. There is a single Internet gateway which is placed in a central position in every topology. The positions of the mesh routers in each topology were generated through a normal distribution over a limited area, using the topology generation algorithm from Machado et al. [43]. This ensures that none of the nodes remain isolated from the network.

\subsection{Results Analysis}

The results obtained in the simulations will be analyzed using confidence interval based on confidence level of 95\% of following performance parameters: throughput, end-to-end delay, path length, and packet loss rate. These parameters are to some extent directly related to each of the objectives that the three approaches (JRCAR, JILP, and MAXI) seek to achieve. This means it will be possible to understand the traffic performance when these routing approaches are taken into account for different numbers of nodes, loads, and interference. The results will be analyzed in the next sub-sections as follows: Sub-section 4.3.1 evaluates the influence of the interference co-channel in the routing approaches. Sub-section 4.3.2 discusses the impact of the external Interference when varying the number of interferer nodes with same Interference Power 
and routing algorithms for every kind of flow in the healthcare monitoring system.

\subsubsection{Co-channel Interference Analysis}

The co-channel interference is caused by the activation of the radio on the nodes themselves for data transmission when the same channel or adjacent channels are using same frequency in a range area [24]. In this context, stations employ a CSMA/CA protocol and will check if the medium is busy or idle. If the medium is busy, they will defer transmission until it is free. Otherwise, they will attempt to transmit data. Co-channel interference has a negative effect on wireless networks when there is an increase in contention. For instance, as wireless devices attempt to use the spectrum in an area of extreme density, they may have to wait for other devices to complete their transmissions.

Thus, there is a need to evaluate the impact of the co-channel interference on the analyzed algorithms. The SpectrumWifiPhy model simulates the wireless network where it is possible to model interference in a more versatile way. This allows to analyze the impact of the co-channel interference on the network. In this subsection, simulation tests are carried out by using the SpectrumWifiPhy as a physical layer model varying the number of nodes in the network and making use of $100 \%$ of active nodes. This analysis aims to assess the impact of different levels of co-channel interference on every routing approach.

Figures 4 and 5 show the Packet Loss Rate (PLR) and Average Flow Throughput (AFT) when the number of nodes in the network is increased. It should also be pointed out that MAXI results in the lowest PLR and highest AFT for high density network (i.e. 40 and 50 nodes) with a very high level of contention and interference. This can be explained by facts that MAXI employs the link quality objective, beyond the two objectives also used in JRCAR and JILP, that minimizes the selection of

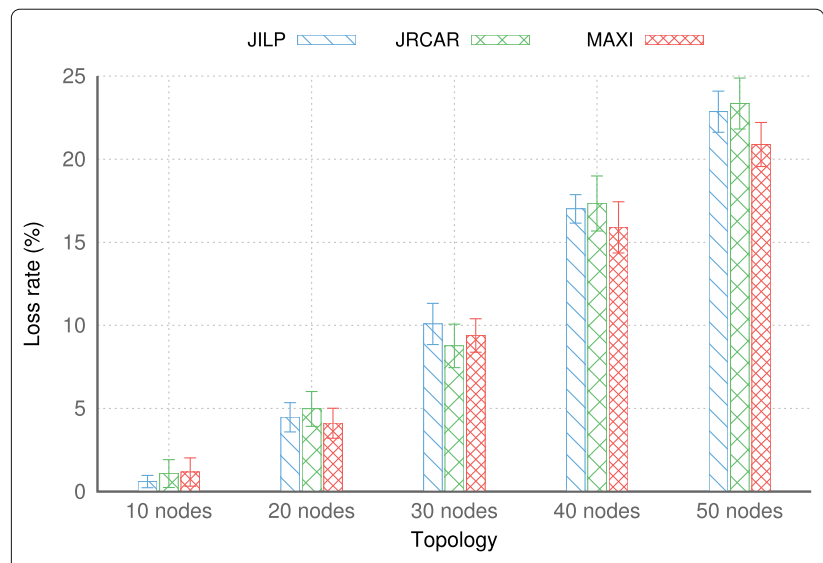

Fig. 4 PLR - packet loss rate varying the quantity of nodes

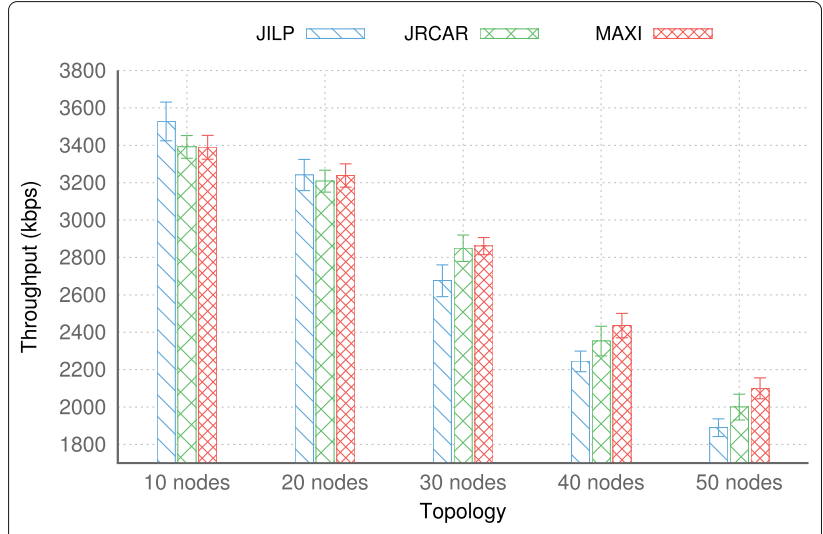

Fig. 5 Throughput varying the quantity of nodes/load

links with high level of interference as well as giving more weigthed to the load balancing objective function in the vital signs application and link quality objective function in fall monitoring application. JILP and JRCAR achieve similar PLR, since they do not take into consideration the link quality function objective.

An alternation between JILP and JRCAR can be observed in throughput (Fig. 5). JILP results in a slightly higher throughput than JRCAR in 10 and 20 nodes, whereas JRCAR achieves the highest throughput in 30, 40 , and 50 nodes because it reaches the lowest delay in packet delivery rates and very similar PLR. This can also be explained by the fact that as the number of nodes/load increases, there is also a rise in the contention level. This means that the routing approaches that result in the shortest paths (JRCAR) improve the throughput by reducing contention levels, since they need a lower number of active links to forward the traffic.

Figure 6 shows the end-to-end delay obtained by the approaches and Fig. 7 shows the average path length (in hops). JRCAR has the lowest average delay, due to the shortest paths achieved by this approach. There are some

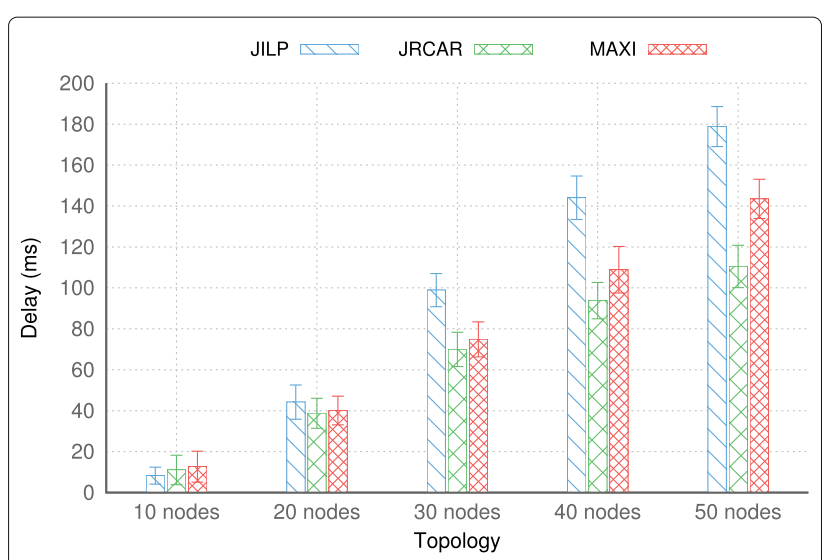

Fig. 6 Delay varying the quantity of nodes 


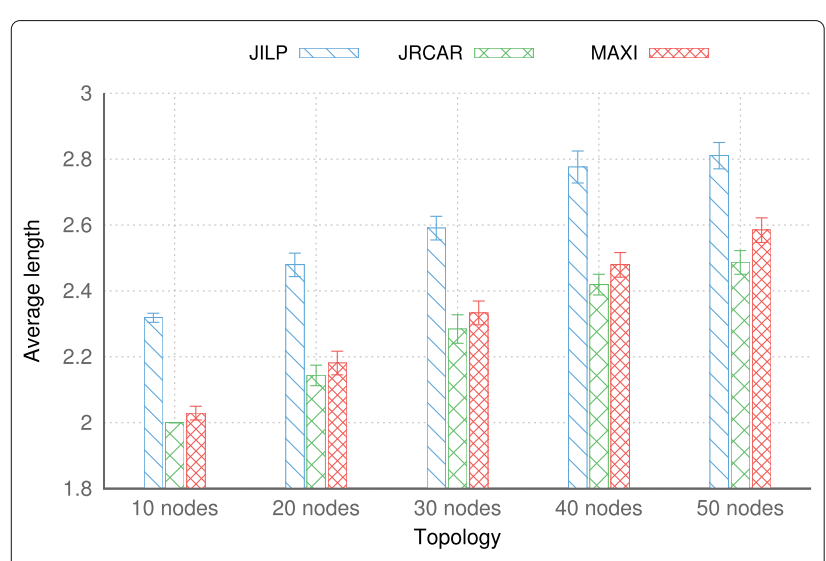

Fig. 7 Average path length varying the quantity of nodes

features that explain the delay caused by JRCAR, the stretch factor used in candidate paths and pre-order of flow calculation based on ascending order (i.e. the routes of nodes with the smallest number of alternatives are firstly calculated) and the tie-breaking criteria based on shorter paths when multiple paths have the same values for bottlenecks. As a result of the priorization of load balancing objective, JILP obtains the highest average path length, and therefore, it achieves the highest average delay. MAXI presents an intermediary average delay since it seeks a trade-off among three weighted objectives. Therefore it achieves slightly higher average delay than JRCAR and slightly lower than JILP.

\subsubsection{Analyzing the External Interference by varying the number of interferer nodes}

Currently, the link quality is the main factor that influences the efficiency of the wireless networks and will continue to do so for some years ahead. Even in the case of IEEE 802.11 standards that still operate in unloaded bands, for instance $5 \mathrm{GHz}$, this issue must be taken into account. This is because the increasing presence of devices with these standards, for instance the IEEE 802.11n and IEEE 802.11ac, will provide greater levels of interference in the future wireless networks that employ these Wi-Fi standards. These devices may have other access points and networks that do not belong to that particular network but by coincidence make use of the same channel. For instance, the LTE-U (LTE-Unlicensed) that proposes the LTE networks use unlicensed spectrum, mainly in $5 \mathrm{Ghz}$ frequency range $[18,44]$. It is important to stress out that transmission power of LTE base stations is much higher than Wi-Fi access point and therefore it causes high level of interference. Hence, these examples will certainly occur in extremely dense scenarios which will become increasingly more common in future networks [45]. This underlines the importance of analyzing the results in a scenario where there is a variation of interference levels within the network.

The SpectrumWiFiPhy model makes it possible to include nodes that create electromagnetic waves at certain frequencies, including Wi-Fi, ZigBee, LTE-U, etc., which means these nodes can be used to simulate the interference caused by devices or even other networks different from WMNs. Simulation tests were carried out to make it easier to assess the impact that the generation of external interference has on the routing approaches, and involved varying the number of nodes that generate external interference from 1 up to 4 nodes. Each node had a power of $0.01 \mathrm{~mW}$ and was positioned at the extreme ends of the simulation area so that each interference node is at the same distance to the Internet gateway, as it is shown in Fig. 8. We evaluated the impact of the generated data on each application listed in Table 5.

The power of the interference is modeled by the Power Spectral Density (PSD), and can be defined as the energy distribution per unit of time in a spectrum frequency. In the NS-3 simulation, the PSD is represented by a set of discrete scalar values for each subband of a determined frequency [42]. This allows the PSD describe how the power of a signal or times series is distributed over a spectrum for continuous signals, such as stationary processes [46]. In this scenario, the interferer node of a spectrum frequency is other devices (access points) employing IEEE $802.11 \mathrm{n}$ or LTE-U. Hence, as the number of interferer nodes increases, there is a rise in the interference level in the network.

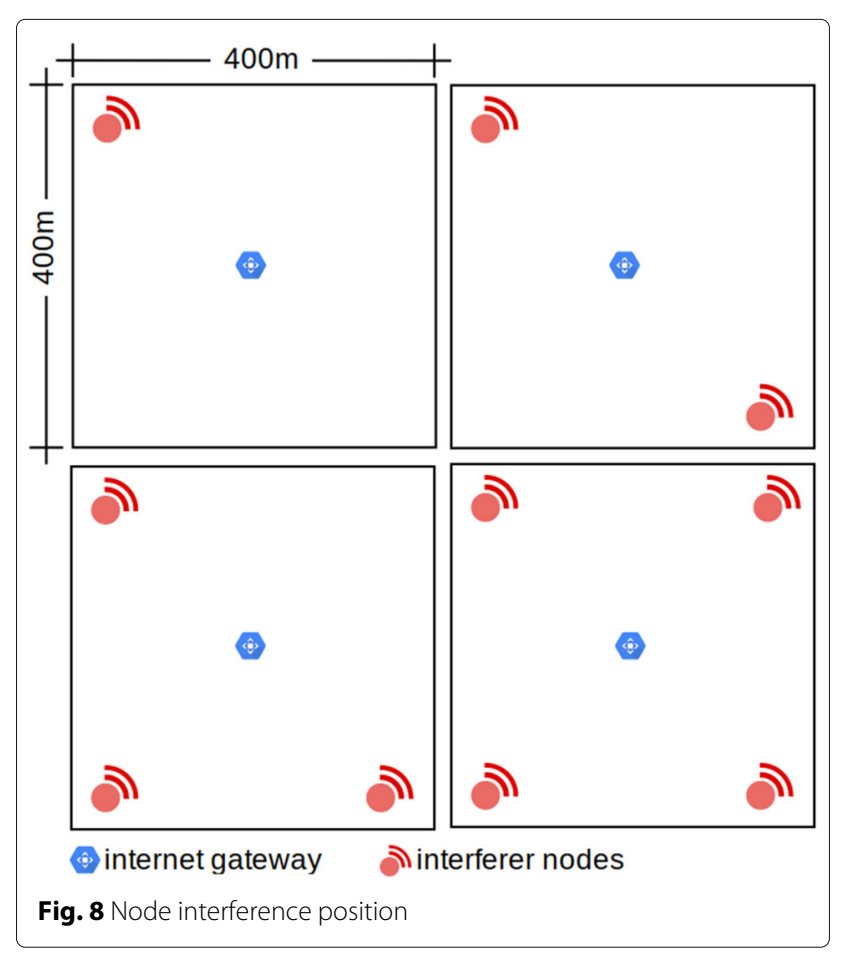




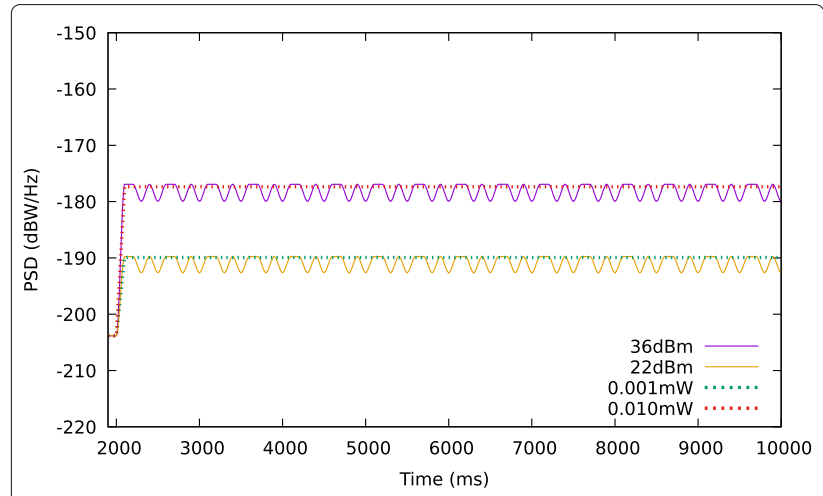

Fig. 9 Transmission power - PSD equivalence between a Wi-Fi node and and interference node of the NS-3 interference generator

As it is shown in Fig. 9, an empirical study of different transmission power values shows that an interference node of the NS-3 interference generator, when transmitting data, using power of $0.01 \mathrm{~mW}$ results in a similar PSD of a Wi-Fi node in the IEEE 802.11n (5Ghz) standard using transmission power about $36 \mathrm{dBm}$. Table 7 shows the packet delivery rate according to the distance of the receiver node, from 250 meters, the delivery rate decreases a lot (smaller than 40\%). It is important to point out that maximum transmission power change according to country, frequency range, and MCS rate. Underdeveloped countries such as Lithuania, Indonesia, Thailand, and South Africa and developed countries such as USA, Canada, China, and Germany usually employ access points with $36 \mathrm{dBm}$ of maximum transmission power [47].

Many people have acquired in the market Wi-Fi signal amplifiers with low cost or changed the maximum transmit power of their routers that can transmit at maximum power without being aware that they may be causing high levels of interference in other WLAN networks in the area.

Table 7 Distance by power reference on NS-3

\begin{tabular}{lll}
\hline distance $(\mathrm{m})$ & $22 \mathrm{dBm}$ & $36 \mathrm{dBm}$ \\
\hline $40 \mathrm{~m}$ & $100.00 \%$ & $100.00 \%$ \\
$60 \mathrm{~m}$ & $100.00 \%$ & $100.00 \%$ \\
$70 \mathrm{~m}$ & $75.00 \%$ & $100.00 \%$ \\
$71 \mathrm{~m}$ & $70.00 \%$ & $100.00 \%$ \\
$73 \mathrm{~m}$ & $27.50 \%$ & $100.00 \%$ \\
$74 \mathrm{~m}$ & $00.00 \%$ & $100.00 \%$ \\
$220 \mathrm{~m}$ & $0.00 \%$ & $100.00 \%$ \\
$250 \mathrm{~m}$ & $0.00 \%$ & $84.62 \%$ \\
$260 \mathrm{~m}$ & $0.00 \%$ & $38.46 \%$ \\
$262 \mathrm{~m}$ & $0.00 \%$ & $25.64 \%$ \\
$263 \mathrm{~m}$ & $0.00 \%$ & $0.00 \%$ \\
\hline
\end{tabular}

Given the size of the simulated scenario area, it is possible to assume that there may be a few nodes that use Wi-Fi access points with this transmission power (approximately $36 \mathrm{dBm}$ ) that can cause considerable interference, especially when considering densely populated areas. For these reasons, a scenario was modeled in this sub-section with some interferer nodes using power of $0.01 \mathrm{~mW}$ positioned at the ends to assess traffic performance basing on different routing solutions with different levels of external interference.

Figure 10 shows the PLR for the three approaches and all applications. MAXI achieves the lowest PLR in most of the configurations and applications, especially when the number of the interferer node increases. These results can be achieved because MAXI assigns significant weights to the load balancing objective function $\left(w_{b}=0.30\right)$ in the vital signs application (Fig. 10a, b, and c) and link quality objective function $\left(w_{l}=0.45\right)$ in fall monitoring application (Fig. 10d and e) employs an objective function. Besides it takes into account of the link quality as objective function. This means that when the links have their quality degraded by the highest number of the interferer nodes, the MAXI approach can find out routes which have a higher link quality, and can thus it achieves a better performance.

JRCAR and JILP fail to take account of the quality of the links in their proposals, which results in a higher packet loss rate. Again, there is an alternation between JILP and JRCAR when varying the number of interferer nodes, JILP reaches lower PLR than JRCAR in one interferer node whereas JRCAR reaches lower PLR for 2, 3, and 4 interferer nodes. Since JILP results in paths with a greater number of hops (as is shown in previous subsection), this increases the chances of it to use low quality links, that reduce the delivery rate of the data. Basing on this fact, MAXI employs high weights for the path length objective function in both vital signs $\left(w_{p}=0.50\right)$ and fall monitoring $\left(w_{p}=0.35\right)$ applications, which also helps to achieve the lowest PLR for both applications with high level of interference (i.e. 3 and 4 interferer nodes). Although MAXI seeks the equilibrium among number of hops, load balancing, and link quality, it gives preference to the objective functions that can take more advantage of specific condition of the scenario to improve the application performance.

Figure 11 shows the AFT for all applications, where the MAXI algorithm achieves the highest AFT in most cases of level of interference and applications (especially when the number of the interferer nodes increases). It is worth noting that MAXI keeps the throughput in a very similar value for all levels of interference, whereas JRCAR and JILP diminish the AFT for Blood Pressure, Depth Sensor, and RGB camera video (Fig. 11a, d, and e) when the interference level increases and better than that, MAXI 


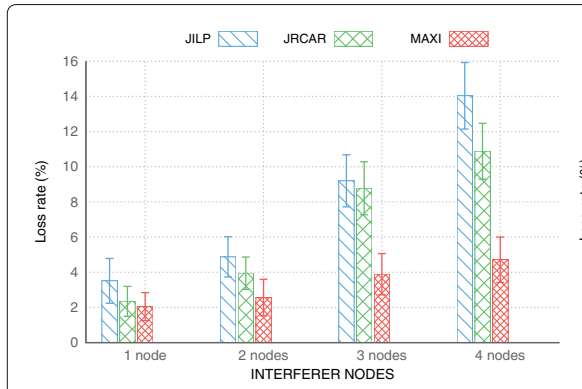

(a)

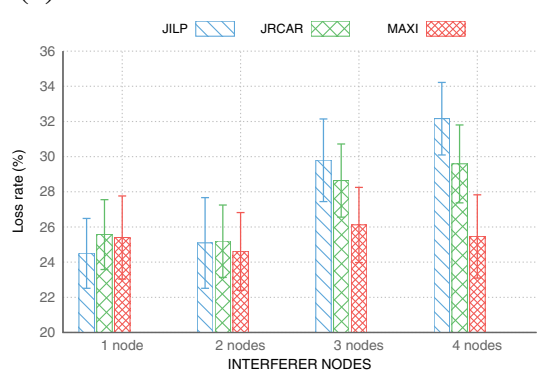

(d)

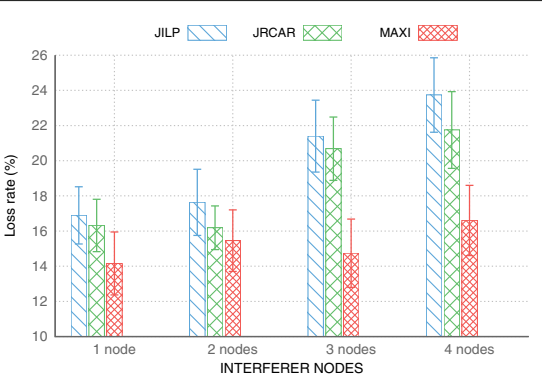

(b)

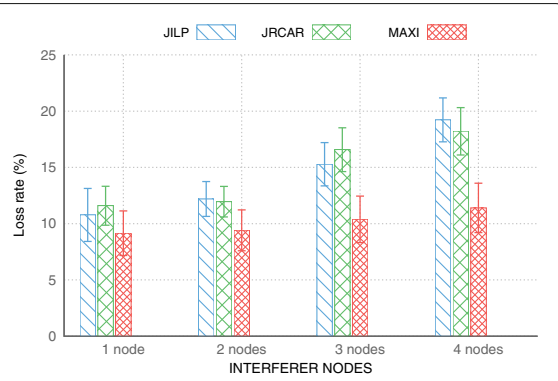

(c)

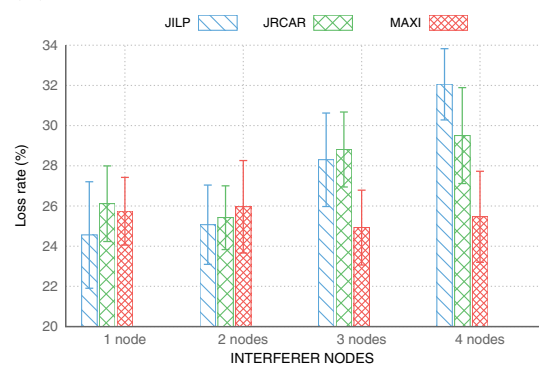

(e)

Fig. 10 Loss rate when varying the number of interferer nodes

increases the AFT in high level of interference (i.e. 3 and 4 interferer nodes) for ECG and RGB camera (Fig. 11b and e) due to fact that link quality objective has the highest weigth $\left(w_{l}=0.45\right)$. As JRCAR and JILP do not take account of the link quality when selecting the routes, the packet loss rate increases which causes a reduction in the throughput, main in JILP.
Figure 12(a), (b), and (c) show the average delay obtained for the flows arising from the vital signs monitoring applications, which needs ultra-low delays due to the urgency of the data delivery, even when there is an increase in the number of interferer nodes. JRCAR results in the lowest delay for most scenarios. However, MAXI results in lower or similar delay compared with JRCAR for some

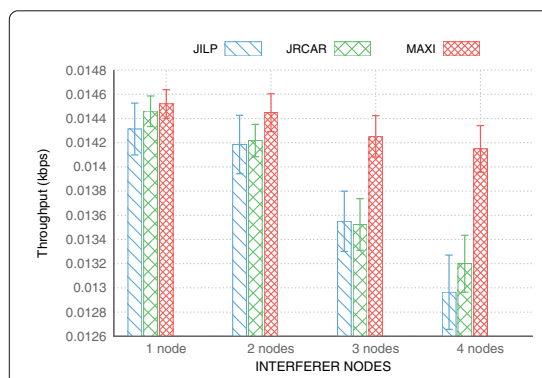

(a)

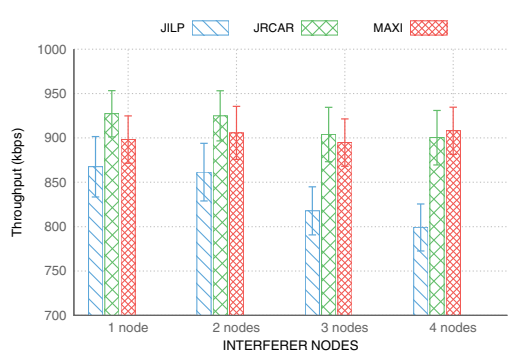

(d)

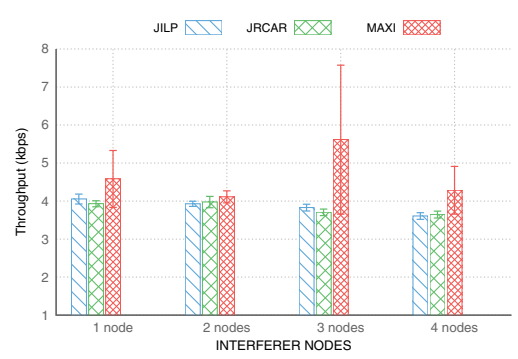

(b)

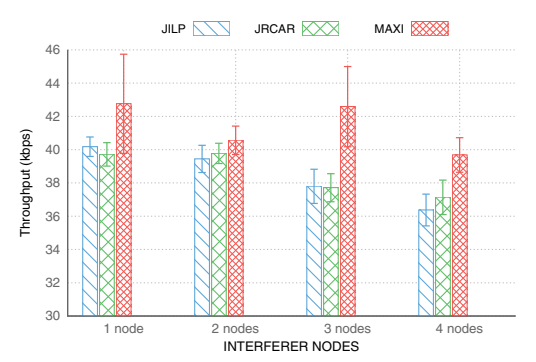

(c)

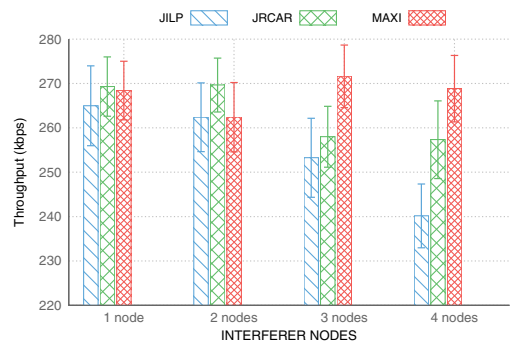

(e)

Fig. 11 Throughput when varying the number of interferer nodes 


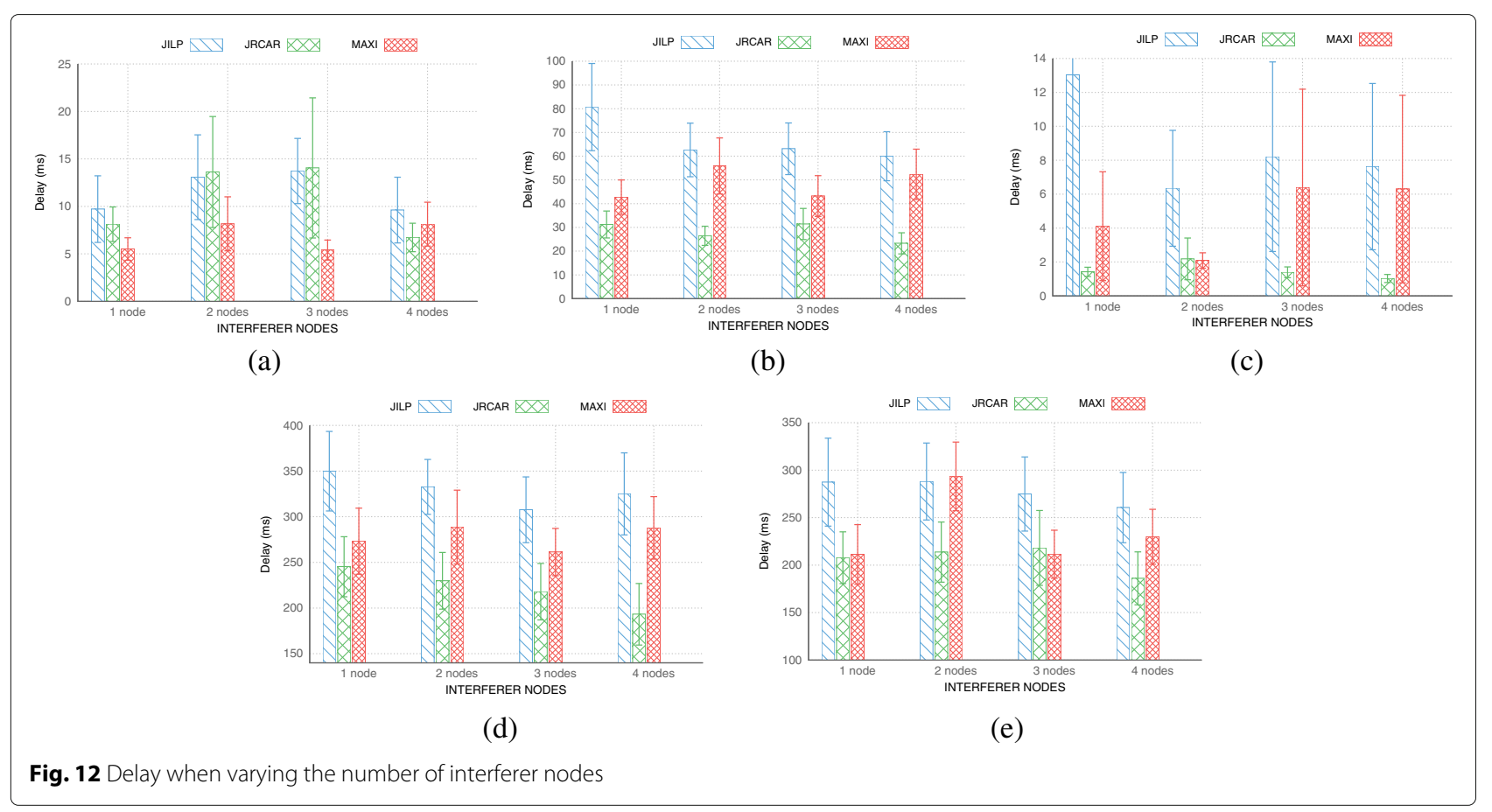

interference levels and the vital signs monitoring applications, because the path length objective employs the highest weight for vital signs monitoring $\left(w_{p}=0.5\right)$.

Different levels of interference directly affect the traffic performance, mainly the delay, through the selected route by all routing approaches. As the number of interferer nodes increases, MAXI seeks to determine routes that avoid the use of regions with high levels of interference. Thus, interference results in a rise in the number of hops, and increases the average end-to-end delay, but not too much when MAXI is employed and moreover, MAXI keeps the delay smaller than the time restriction (125ms). It is important to stress out only the successful transmission packets are taken into account for the delay calculation. JRCAR results in higher PLR and lower AFT than MAXI for most interference levels and applications, thus the end-to-end delay can not be too much precise parameter of traffic performance for all routing approaches.

Besides MAXI results in more constant loss rate and throughput when increasing interference and traffic load, the proposal show statistically significant difference, based on the 2-Sample T test [48], with the second best approach (JILP or JRCAR) in throughput and loss rate when there are high levels of external interference (i.e. 3 and 4 interferer nodes). In a confidence level of 95\%, the 2-Sample $\mathrm{T}$ test evidenced a significant difference between the two samples (MAXI and the best second approach (JILP or JRCAR)) for the most network configurations, due the resultant $P$-value, as it is shown in Table 8 when $P$-value $<0.05$, it indicates a significant difference between two samples. In addition, MAXI enables much lesser loss rate (50\%, on average of all applications) and higher throughput.

\section{Conclusion and Suggestion for Future Works}

There is a lacking of routing solutions to support different requirements of mixed traffic for future IoT applications. In this article, we set to cover this gap by introducing a more efficient multi-objetive routing ILP model and algorithm, called MAXI, which maximizes the overall mixed traffic performance through supporting the requirements of each application in mixed IoT system. In addition, this article has carried out an in-depth and detailed study of comparison between the main multi-objective routing algorithms for WMNs based on the ILP model, e.g. JRCAR and JILP. The current and future IoT system (monitoring for the elderly) with different kinds of applications (camera video RGB, sensor depth, ECG, blood pressure, etc.) and different types of interference (co-channel interference and external interference) were assessed in a simulated scenario.

The results show that the distinct kind and levels of the interference has impact on resulting traffic performance achieving by all routing approaches, nonetheless MAXI outperforms JRCAR and JILP in terms of most performance parameters and scenario configurations, mostly because it employs the weighted sum of three important function objectives according to distinct requirements of each application. It is worth noting that the throughput 
Table 8 2-Sample T test between MAXI and the second best approach (JILP or JRCAR)

\begin{tabular}{|c|c|c|c|c|c|c|c|}
\hline \multirow[b]{2}{*}{ Result } & \multirow[b]{2}{*}{ Figure } & \multicolumn{2}{|l|}{ MAXI } & \multicolumn{2}{|c|}{ Second Best Approach } & \multirow[b]{2}{*}{$P$-value } & \multirow[b]{2}{*}{ Approach } \\
\hline & & Mean & Std. Dev. & Mean & Std. Dev. & & \\
\hline PLR Packet Loss Rate 50 node. & Figure 4 & $20.88 \%$ & 4.1777 & $22.85 \%$ & 3.7374 & 0.0291798 & JILP \\
\hline Throughput - (kbps) 50 nodes & Figure 5 & 2099.60 & 176.7813 & 1999.80 & 214.1429 & 0.0258763 & JRCAR \\
\hline PLR Packet Loss Rate 3 interferer nodes & Figure 10.a & $3.88 \%$ & 3.64508 & $8.77 \%$ & 4.3487 & $6.01526 \mathrm{e}-7$ & JRCAR \\
\hline PLR Packet Loss Rate 4 interferer nodes & Figure 10.a & $4.71 \%$ & 3.79077 & $10.87 \%$ & 4.7405 & $1.12835 \mathrm{e}-8$ & JRCAR \\
\hline PLR Packet Loss Rate 3 interferer nodes & Figure 10.b & $14.73 \%$ & 6.0360 & $20.68 \%$ & 5.1981 & 0.000010434 & JRCAR \\
\hline PLR Packet Loss Rate 4 interferer nodes & Figure 10.b & $16.59 \%$ & 5.8581 & $21.74 \%$ & 6.4999 & 0.000375204 & JRCAR \\
\hline PLR Packet Loss Rate 3 interferer nodes & Figure 10.c & $10.38 \%$ & 6.3970 & $15.27 \%$ & 5.8087 & 0.0005940690 & JILP \\
\hline PLR Packet Loss Rate 4 interferer nodes & Figure 10.c & $11.39 \%$ & 6.4491 & $18.20 \%$ & 6.2584 & $0.0771206 \mathrm{e}-4$ & JRCAR \\
\hline PLR Packet Loss Rate 3 interferer nodes & Figure 10.d & $26.11 \%$ & 6.6300 & $28.63 \%$ & 6.0063 & $0.078334^{*}$ & JRCAR \\
\hline PLR Packet Loss Rate 4 interferer nodes & Figure 10.d & $25.46 \%$ & 6.9406 & $29.58 \%$ & 6.5963 & 0.0078889 & JRCAR \\
\hline PLR Packet Loss Rate 3 interferer nodes & Figure 10.e & $24.92 \%$ & 5.7788 & $28.29 \%$ & 7.0203 & 0.0214786 & JILP \\
\hline PLR Packet Loss Rate 4 interferer nodes & Figure 10.e & $25.46 \%$ & 6.6399 & $29.50 \%$ & 7.0977 & 0.0103543 & JRCAR \\
\hline Throughput - (kbps) 3 interferer nodes & Figure 11.a & 0.0142 & 0.0005 & 0.0129 & 0.0007 & $4.73843 e-13$ & JILP \\
\hline Throughput - (kbps) 4 interferer nodes & Figure 11.a & 0.0141 & 0.0005 & 0.0132 & 0.0007 & $3.87685 \mathrm{e}-9$ & JRCAR \\
\hline Throughput - (kbps) 3 interferer nodes & Figure 11.b & 5.6153 & 7.4809 & 3.8274 & 3.0998 & $0.168543^{*}$ & JILP \\
\hline Throughput - (kbps) 4 interferer nodes & Figure 11.b & 4.2828 & 1.8386 & 3.6457 & 0.2723 & 0.0360313 & JRCAR \\
\hline Throughput - (kbps) 3 interferer nodes & Figure 11.c & 42.5862 & 6.0842 & 37.7976 & 0.2702 & 0.00001364 & JILP \\
\hline Throughput - (kbps) 4 interferer nodes & Figure 11.c & 39.6792 & 3.0428 & 37.1380 & 3.0790 & 0.00038309 & JRCAR \\
\hline Throughput - (kbps) 3 interferer nodes & Figure 11.d & 894.7601 & 82.7290 & 903.9110 & 88.4366 & $0.634047^{*}$ & JRCAR \\
\hline Throughput - (kbps) 4 interferer nodes & Figure 11.d & 908.0184 & 91.2944 & 900.2777 & 78.0516 & $0.684719^{*}$ & JRCAR \\
\hline Throughput - (kbps) 3 interferer nodes & Figure 11.e & 271.6090 & 21.8762 & 258.0036 & 19.8047 & 0.0046429 & JRCAR \\
\hline Throughput - (kbps) 4 interferer nodes & Figure 11.e & 268.8379 & 21.9425 & 257.3389 & 26.0504 & 0.0359653 & JRCAR \\
\hline
\end{tabular}

* samples with a $P$-value $\geq 0.05$

and loss rate are improved significantly when MAXI is employed in network configurations with high levels of external and co-channel interference. In future work, this article will raise an open issue that needs attention, i.e. how to adapt dynamically the weights for mixed IoT traffic based on regular and sporadic data and, the $T_{h}$ and $T_{l}$ thresholds of $I R$.

\subsection{Source codes}

All source codes for the algorithms used in this article are at the following link:: https://github.com/vnmedeiros/ MAXI.

\section{Acknowledgments}

This work has been partially supported by the CAPES (Brazil).

\section{Funding}

CAPES provided a grant scholarhip during this reseach development. CAPES is a Brazilian government agency awarding scholarship grants to graduate and posgraduate students at universities and research centers.

\section{Availability of data and materials}

Not applicable.

\section{Authors' contributions}

VN proposed and developed the work and, carried out the simulation assessment, wrote the article. BS was the co-advisor that help to define the problem and review the article suggesting a lot of content and corrections to the article. VB was the advisor that defined the initial problem and make many suggestions to improve the research and this article. All authors read and approved the final manuscript.

Competing interests

The authors declare that they have no competing interests.

\section{Publisher's Note}

Springer Nature remains neutral with regard to jurisdictional claims in published maps and institutional affiliations.

Received: 20 November 2018 Accepted: 4 March 2019

Published online: 01 May 2019

\section{References}

1. Mainwaring A, Culler D, Polastre J, Szewczyk R, Anderson J. Wireless sensor networks for habitat monitoring. In: Proceedings of the 1st ACM International Workshop on Wireless Sensor Networks and Applications. New York: ACM; 2002. p. 88-97.

2. Cerpa A, Elson J, Estrin D, Girod L, Hamilton M, Zhao J. Habitat monitoring: Application driver for wireless communications technology. ACM SIGCOMM Comput Commun Rev. 2001;31(2 supplement):20-41.

3. Oliveira $L$, Rodrigues J. Wireless sensor networks: a survey on environmental monitoring. J Commun. 2011;6(2):143-51.

4. Alanazi A, Elleithy K. Real-time QoS routing protocols in wireless multimedia sensor networks: Study and analysis. Sensors. 2015;15(9): 22209. https://doi.org/10.3390/s150922209.

5. Atzori L, lera A, Morabito $G$. The Internet of Things: A survey. Comput Netw. 2010;54(15):2787-805. 
6. Werner-Allen G, Johnson J, Ruiz M, Lees J, Welsh M. Monitoring volcanic eruptions with a wireless sensor network. In: Proceeedings of the Second European Workshop on Wireless Sensor Networks. Istanbul: IEEE; 2005 p. 108-20.

7. Malan D, Fulford-Jones T, Welsh M, Moulton S. Codeblue: An ad hoc sensor network infrastructure for emergency medical care. In: International Workshop on Wearable and Implantable Body Sensor Networks, vol. 5. London: dash harvard; 2004

8. Gao T, Pesto C, Selavo L, Chen Y, Ko J, Lim J, Terzis A, Watt A, Jeng J, Chen B.-r., et al. Wireless medical sensor networks in emergency response: Implementation and pilot results. In: Technologies for Homeland Security, IEEE Conference on. Boston: IEEE; 2008. p. 187-92.

9. Burrell J, Brooke T, Beckwith R. Vineyard computing: Sensor networks in agricultural production. IEEE Pervasive Comput. 2004;3(1):38-45.

10. Ruiz-Garcia L, Lunadei L, Barreiro P, Robla I. A review of wireless sensor technologies and applications in agriculture and food industry: State of the art and current trends. Sensors. 2009;9(6):4728-50.

11. Floris A, Atzori L. Managing the quality of experience in the multimedia internet of things: A layered-based approach. Sensors. 2016;16(12). https://doi.org/10.3390/s16122057.

12. Akyildiz IF, Nie S, Lin S-C, Chandrasekaran M. 5 g roadmap: 10 key enabling technologies. Comput Netw. 2016;106:17-48.

13. Wang C, Haider F, Gao X, You X, Yang Y, Yuan D, Aggoune HM, Haas H, Fletcher S, Hepsaydir E. Cellular architecture and key technologies for $5 \mathrm{~g}$ wireless communication networks. IEEE Commun Mag. 2014;52(2):122-30.

14. Hossain E, Rasti M, Tabassum H, Abdelnasser A. Evolution toward $5 \mathrm{~g}$ multi-tier cellular wireless networks: An interference management perspective. IEEE Wirel Commun. 2014;21(3):118-27.

15. Osseiran A, Boccardi F, Braun V, Kusume K, Marsch P, Maternia M, Queseth O, Schellmann M, Schotten H, Taoka H, Tullberg H, Uusitalo MA Timus B, Fallgren M. Scenarios for $5 \mathrm{~g}$ mobile and wireless communications: the vision of the metis project. IEEE Commun Mag. 2014;52(5):26-35.

16. Jurdi R, Gupta AK, Andrews JG, Heath RW. Modeling infrastructure sharing in mmwave networks with shared spectrum licenses. IEEE Trans Cogn Commun Netw. 2018;4(2):328-43.

17. Zhou Z, Mumtaz S, Huq KMS, Al-Dulaimi A, Chandra K, Rodriquez J. Cloud miracles: Heterogeneous cloud ran for fair coexistence of Ite-u and wi-fi in ultra dense $5 \mathrm{~g}$ networks. IEEE Commun Mag. 2018;56(6):64-71.

18. Bocanegra C, Kennouche TE, Li Z, Favalli L, Felice MD, Chowdhury KR. E-fi: Evasive wi-fi measures for surviving Ite within $5 \mathrm{ghz}$ unlicensed band. IEEE Trans Mob Comput. 2018;18(4):830-44.

19. Feng L, Zhao P, Zhou F, Yin M, Yu P, Li W, Qiu X. Resource allocation for $5 \mathrm{~g} \mathrm{~d} 2 \mathrm{~d}$ multicast content sharing in social-aware cellular networks. IEEE Commun Mag. 2018;56(3):112-8.

20. Akyildiz IF, Wang X, Wang W. Wireless mesh networks: a survey. Comput Netw. 2005;47(4):445-87.

21. Gálvez JJ, Ruiz PM. Efficient rate allocation, routing and channel assignment in wireless mesh networks supporting dynamic traffic flows. Ad Hoc Netw. 2013;11(6):1765-81. https://doi.org/10.1016/j.adhoc.2013.04.002.

22. de Mello MOMC, Borges VCM, Pinto LL, Cardoso KV. Improving load balancing, path length, and stability in low-cost wireless backhauls. Ad Hoc Netw. 2016;48:16-28. https://doi.org/10.1016/j.adhoc.2016.05.002.

23. Medeiros VN, de Brito PCG, Silvestre B, da CM Borges V. Rall: Routing-aware of path length, link quality and traffic load for wireless sensor networks. In: Proceedings of the Symposium on Applied Computing. SAC '17. New York: ACM; 2017. p. 594-601. https://doi.org/ 10.1145/3019612.3019730. http://doi.acm.org/10.1145/3019612.3019730. https://dl.acm.org/citation.cfm?id=3019730.

24. Boushaba M, Hafid A, Gendreau M. Source-based routing in wireless mesh networks. IEEE Syst J. 2016;10(1):262-70.

25. Matos R, Coutinho N, Marques C, Sargento S, Chakareski J, Kassler A Quality of experience-based routing in multi-service wireless mesh networks. In: 2012 IEEE International Conference on Communications (ICC); 2012. p. 7060-5. https://doi.org/10.1109/ICC.2012.6364944.

26. Hao Z, Li Y. An adaptive load-aware routing algorithm for multi-interface wireless mesh networks. Wirel Netw. 2015;21(2):557-64.

27. Alwan NAS. Performance analysis of dijkstra-based weighted sum minimization routing algorithm for wireless mesh networks. Model Simul Eng. 2014;2014:32-323232. https://doi.org/10.1155/2014/658408.
28. Lozano-Garzon C, Camelo M, Vila P, Donoso Y. A multi-objective routing algorithm for wireless mesh network in a smart cities environment. J Netw. 2015;10(01):60-9.

29. Jain K, Padhye J, Padmanabhan VN, Qiu L. Impact of interference on multi-hop wireless network performance. Wirel Netw. 2005;11(4):471-87. https://doi.org/10.1007/s11276-005-1769-9.

30. Borges VCM, Curado M, Monteiro E. Cross-layer routing metrics for mesh networks: Current status and research directions. Comput Commun. 2011;34(6):681-703.

31. Sobrinho JL. Algebra and algorithms for qos path computation and hop-by-hop routing in the internet. IEEE/ACM Trans Networking. 2002;10(4):541-50.

32. Zhu N, Diethe T, Camplani M, Tao L, Burrows A, Twomey N, Kaleshi D, Mirmehdi M, Flach P, Craddock I. Bridging e-health and the internet of things: The sphere project. IEEE Intell Syst. 2015;30(4):39-46. https://doi. org/10.1109/MIS.2015.57.

33. Kolodzey L, Grantcharov PD, Rivas H, Schijven MP, Grantcharov TP. Wearable technology in the operating room: a systematic review. BMJ Innov. 2016. https://doi.org/10.1136/bmjinnov-2016-000133. http:// innovations.bmj.com/content/early/2016/12/21/bmjinnov-2016-000133. full.pdf.

34. Soh PJ, Vandenbosch GAE, Mercuri M, Schreurs DMMP. Wearable wireless health monitoring: Current developments, challenges, and future trends. IEEE Microw Mag. 2015;16(4):55-70. https://doi.org/10. 1109/MMM.2015.2394021.

35. Acampora G, Cook DJ, Rashidi P, Vasilakos AV. A survey on ambient intelligence in healthcare. Proc IEEE. 2013;101(12):2470-94.

36. Chan M, Estève D, Fourniols J-Y, Escriba C, Campo E. Smart wearable systems: Current status and future challenges. Artif Intell Med. 2012;56(3): 137-56. https://doi.org/10.1016/j.artmed.2012.09.003.

37. Zhu Z, Liu T, Li G, LiT, Inoue Y. Wearable sensor systems for infants. Sensors. 2015;15(2):3721-49. https://doi.org/10.3390/s150203721.

38. Movassaghi S, Abolhasan M, Lipman J, Smith D, Jamalipour A. Wireless body area networks: A survey. IEEE Commun Surv Tutor. 2014;16(3): 1658-86. https://doi.org/10.1109/SURV.2013.121313.00064.

39. Latré B, Braem B, Moerman I, Blondia C, Demeester P. A survey on wireless body area networks. Wirel Netw. 2011;17(1):1-18. https://doi.org/ 10.1007/s11276-010-0252-4.

40. Hayajneh T, Almashaqbeh G, Ullah S, Vasilakos AV. A survey of wireless technologies coexistence in wban: analysis and open research issues. Wirel Netw. 2014;20(8):2165-99. https://doi.org/10.1007/s11276-0140736-8.

41. Lacage $M$, Henderson TR. Yet another network simulator. In: Proceeding from the 2006 Workshop on Ns-2: The IP Network Simulator. WNS2 '06. New York: ACM; 2006. https://doi.org/10.1145/1190455.1190467. http:// doi.acm.org/10.1145/1190455.1190467.

42. Baldo N, Miozzo M. Spectrum-aware channel and phy layer modeling for ns3. In: Proceedings of the Fourth International ICST Conference on Performance Evaluation Methodologies and Tools. VALUETOOLS '09. ICST, Brussels: ICST (Institute for Computer Sciences, Social-Informatics and Telecommunications Engineering); 2009. p. 2-128. https://doi.org/10. 4108/ICST.VALUETOOLS2009.7647.

43. Machado K, Rosário D, Cerqueira E, Loureiro AAF, Neto A, de Souza JN. A routing protocol based on energy and link quality for internet of things applications. Sensors. 2013;13(2):1942. https://doi.org/10.3390/ s130201942.

44. Tariq M, Anjum MR, Amjad M. Design of simulation system for Ite-u using 5 ghz band in matlab. Wirel Pers Commun. 2018;100(4):1661-76.

45. Borges VCM, Cardoso KV, Cerqueira E, Nogueira M, Santos A. Aspirations, challenges, and open issues for software-based $5 \mathrm{~g}$ networks in extremely dense and heterogeneous scenarios. EURASIP J Wirel Commun Netw. 2015;2015(1):164. https://doi.org/10.1186/s13638-015-0380-8.

46. Martin R. Noise power spectral density estimation based on optimal smoothing and minimum statistics. IEEE Trans Speech Audio Process. 2001;9(5):504-12.

47. Cisco. Cisco: Supported Country Codes for Wireless LAN. 2018. https:// www.cisco.com/c/en/us/td/docs/wireless/wcs/3-2/configuration/guide/ wcscfg32/wcscod.pdf. Accessed 28 Nov 2015.

48. Montgomery DC. Design and Analysis of Experiments. USA: Wiley; 2006. 